Draft VERSion SEPTEMBer 26, 2018

Preprint typeset using $\mathrm{LT}_{\mathrm{E}} \mathrm{X}$ style emulateapj v. 5/2/11

\title{
DECONSTRUCTING THE KINETIC SZ POWER SPECTRUM
}

\author{
Laurie D. ShaW ${ }^{1,2}$, Douglas H. RudD ${ }^{1,2}$, And Daisuke Nagai ${ }^{1,2}$ \\ Draft version September 26, 2018
}

\begin{abstract}
We present a detailed investigation of the impact of astrophysical processes on the shape and amplitude of the kinetic SZ (kSZ) power spectrum from the post-reionization epoch. This is achieved by constructing a new model of the kSZ power spectrum which we calibrate to the results of hydrodynamic simulations. By construction, our method accounts for all relevant density and velocity modes and so is unaffected by the limited box size of our simulations. We find that radiative cooling and star-formation can reduce the amplitude of the $\mathrm{kSZ}$ power spectrum by up to $33 \%$, or $1 \mu \mathrm{K}^{2}$ at $\ell=3000$. This is driven by a decrease in the mean gas density in groups and clusters due to the conversion of gas into stars. Variations in the redshifts at which helium reionization occurs can effect the amplitude by a similar fraction, while current constraints on cosmological parameters (namely $\sigma_{8}$ ) translate to a further $\pm 15 \%$ uncertainty on the $\mathrm{kSZ}$ power spectrum. We demonstrate how the models presented in this work can be constrained - reducing the astrophysical uncertainty on the kSZ signal - by measuring the redshift dependence of the signal via kSZ tomography. Finally, we discuss how the results of this work can help constrain the duration of reionization via measurements of the kinetic SZ signal sourced by inhomogeneous (or patchy) reionization.
\end{abstract}

Subject headings: cosmology: cosmic microwave background - cosmology: large-scale structure of universe - galaxies: clusters: general — intergalactic medium

\section{INTRODUCTION}

In the last few years, significant progress has been made towards making precision measurements of the temperature anisotropy in the cosmic microwave background (CMB) at sub-degree scales. Ground-based experiments such as the South Pole Telescope (Ruhl et al. 2004) and the Atacama Cosmology Telescope (Kosowsky 2003) have achieved the necessary combination of sensitivity and angular resolution to measure the primary CMB temperature anisotropy power spectrum to the seventh acoustic peak and beyond (Dunkley et al. 2011; Keisler et al. 2011). The Planck satellite will measure the CMB temperature power spectrum at cosmicvariance limited precision to angular scales below one tenth of a degree.

On angular scales smaller than $\sim 4$ arcminutes $(\ell \gtrsim$ 2700 ), the CMB power spectrum is dominated by 'secondary' anisotropies, temperature fluctuations that are generated by the interaction of CMB photons with large scale structure between the observer and the surface of last scattering. The principal contribution to the secondary anisotropy signals comes from the SunyaevZel'dovich (SZ) effect, which can be broken down into two components- the 'thermal' and 'kinetic' effects. The former describes the transfer of thermal energy from free electrons in the hot intra-cluster medium to CMB photons via inverse-Compton scattering. CMB photons receive a boost in energy, distorting the Planckian form of its spectrum. This gives the thermal SZ (tSZ) effect a unique frequency dependence which can be utilized by

\footnotetext{
${ }^{1}$ Department of Physics, Yale University, New Haven, CT 06520

${ }^{2}$ Yale Center for Astronomy \& Astrophysics, Yale University, New Haven, CT 06520

laurie.shaw@yale.edu

3 http://www.rssd.esa.int/index.php?project=Planck
}

experiments to extract this signal from the primary CMB signal and foregrounds. The kinetic SZ (kSZ) effect is caused by the doppler shifting of CMB photons via scattering off clouds of electrons with a non-zero bulk velocity (along the line-of-sight) relative to the CMB rest frame. Unlike the tSZ effect, the $\mathrm{kSZ}$ has the same frequency dependence as the primary CMB.

The thermal SZ effect has now been detected for both large numbers of individual clusters (Vanderlinde et al. 2010; Marriage et al. 2011; Williamson et al. 2011; Planck Collaboration et al. 2011; Marrone et al. 2011) and as a secondary anisotropy signal in the CMB power spectrum (Lueker et al. 2010; Dunklev et al.|2011; Shirokoff et al. 2011). While the kSZ effect has not yet been detected in either case, it is likely be done so first in the power spectrum. CMB temperature fluctuations sourced by the kSZ effect are proportional to the product of the electron density and line-of-sight velocity. The lack of an electron temperature weighting means that the contribution of low-temperature gas is more significant than for the tSZ effect (which is proportional to the product of electron density and temperature). On the other hand, the kSZ signal from individual groups and clusters is weaker.

The kSZ power spectrum is also sensitive to the details of reionization (Gruzinov \& Hu 1998; Knox et al. 1998; McQuinn et al. 2005; Zahn et al.|2005; Iliev et al. 2007). In models of inhomogeneous or patchy reionization - in which different regions of the Universe are reionized at different times - bubbles of free electrons around UVemitting sources are embedded in an otherwise neutral medium. If these bubbles have a large-scale bulk velocity then they will impart a temperature anisotropy onto the CMB. In this work we refer to the $\mathrm{kSZ}$ effect from reionization as the 'patchy' signal, while that from epochs after reionization is complete is referred to as the 
'post-reionization' signal.

Zahn et al. (2005) and McQuinn et al. (2005) demonstrated that, to first order, the magnitude of the $\mathrm{kSZ}$ power from patchy reionization is dependent on the duration of reionization. Hence, when combined with measurements of the optical depth to reionization from the primary CMB power spectrum, the redshift range spanned by the epoch of reionization can be constrained. For example, if reionization started at $z=14$ and ended at $z=6$, it would generate roughly $3 \mu \mathrm{K}^{2}$ of patchy $\mathrm{kSZ}$ power (at $\ell=3000$ ), while the range $8 \leq z \leq 12$ would generate $1.5 \mu \mathrm{K}^{2}$ (McQuinn et al. 2005). While there are several methods for probing the redshift at which reionization ended (e.g., Oh \& Furlanetto 2005; Fan et al. 2006; Lidz et al.|2006; Becker et al.|2007; Bolton \& Haehnelt 2007; Ouchi et al. 2010; Mortlock et al. 2011, and references therein), there are currently no other means by which the duration of reionization can be measured, making the $\mathrm{kSZ}$ power spectrum a unique and exciting probe.

The patchy $\mathrm{kSZ}$ signal is expected to have a slightly different angular shape to the post-reionization signal, peaking at a larger angular scale $(\ell \approx 2000$, Zahn et al. 2005, 2011), corresponding to the characteristic bubble size during reionization. In principle, the two components of the $\mathrm{kSZ}$ signal can be separated by a precise measurement of the power spectrum that encompasses a wide range of angular scales. In practice, however, the primary CMB signal swamps that of the $\mathrm{kSZ}$ at $\ell<3000$, while extra-galactic foregrounds dominate at $\ell>5000$. Hence, differentiating the post-reionization kSZ power from that sourced by patchy reionization via their angular scale dependence is an intractable task. To measure the patchy component of this signal it is therefore vitally important to have a good theoretical understanding of the post-reionization contribution.

One of the principal aims of this work is to investigate the theoretical uncertainty in the post-reionization $\mathrm{kSZ}$ power spectrum. Specifically, we aim to investigate the impact of astrophysical processes such as radiative cooling of gas, the formation of stars and galaxies and feedback from supernovae. We construct an analytic model for the $\mathrm{kSZ}$ power spectrum which we calibrate to the results of hydrodynamic simulations. These simulations are run in both the non-radiative regime, and including radiative cooling and star-formation. We are thus able to compare the impact of these processes on the $\mathrm{kSZ}$ power spectrum. Our model also enables us to investigate the cosmological scaling of the kSZ power spectrum.

Several previous studies have measured the kSZ power spectrum directly from cosmological simulations by generating synthetic sky maps, projecting through the simulation box stacked over multiple timesteps (White et al. 2002; Hallman et al. 2009; Battaglia et al. 2010). The principal drawback to this approach is that very large simulation box-sizes are necessary to adequately capture the large-scale velocity flows that contribute significantly to the kSZ signal at small angular scales (Zhang et al. 2004). As we shall demonstrate, simulations with box sizes significantly less than $1 \mathrm{Gpc} / h$, systematically and substantially underestimate the $\mathrm{kSZ}$ power spectrum. Furthermore, high spatial resolution is required to resolve small-scale baryonic processes such as cooling and star-formation.

In this work we adopt a hybrid approach. We use highresolution simulations to capture the effect of non-linear structure formation on the gas density power spectrum, and use the results to improve our analytic calculation for the kSZ effect. Our method accounts for all relevant velocity and density modes, and circumvents the prohibitive requirement of high spatial resolution and extremely large cosmological boxes.

This paper is organized as follows: in Section 2 we provide an overview of the $\mathrm{kSZ}$ effect, describe our model for the $\mathrm{kSZ}$ power spectrum, and discuss how we implement the modifications required to account for the astrophysical processes in our simulations. In Section 3 we describe the hydrodynamic simulations used to calibrate our model. In Section 4 we present results from the simulations, focusing specifically on the gas density and momentum power spectra and comparing these with the predictions of our models. In Section 5 we discuss the $\mathrm{kSZ}$ power spectra predicted by our models, their scaling with cosmological parameters, and the means by which they could be distinguished observationally. We also investigate the impact of helium reionization on the power spectrum. Finally, we compare our model with the results of previous work and the latest observations.

Except when referring to specific simulations with specific cosmological parameters, throughout this paper we assume a fiducial, spatially-flat, $\Lambda \mathrm{CDM}$ cosmological model consistent with the WMAP7 best-fit cosmological parameters, namely $H_{0}=71 \mathrm{~km} \mathrm{~s}^{-1} \mathrm{Mpc}^{-1}, \Omega_{M}=0.27$, $\Omega_{b}=0.047, \Omega_{\Lambda}=0.73, n_{s}=0.95$ and $\sigma_{8}=0.82$.

\section{MODELING THE KINETIC SZ POWER SPECTRUM}

\section{1. kSZ Basics}

Thomson scattering of CMB photons off clouds of free electrons with a coherent bulk velocity along the lineof-sight from the observer produce fluctuations in the observed brightness temperature of the CMB,

$$
\frac{\Delta T}{T_{\mathrm{cmb}}}(\hat{\mathbf{n}})=\frac{\sigma_{T}}{c} \int_{0}^{z_{\mathrm{rei}}} \frac{d x}{d z} \frac{d z}{(1+z)} \exp (-\tau(z)) n_{e}(z) \mathbf{v} \cdot \hat{\mathbf{n}},
$$

where $\sigma_{T}$ is the Thomson cross-section for an electron, $x$ is the comoving distance to redshift $z, n_{e}$ is the free electron number density and $\mathbf{v} \cdot \hat{\mathbf{n}}$ is the component of the peculiar velocity of the electrons along the line-ofsight. We are principally concerned with the kinetic SZ power spectrum in the post-reionization era, so the upper limit to the integral, $z_{\text {rei }}$, corresponds to the redshift at which reionization ends (i.e., hydrogen has been entirely ionized). Unless stated otherwise we assume a fiducial value of $z_{\text {rei }}=10$.

$\tau$ is the Thomson optical depth,

$$
\tau(z)=\sigma_{T} \int_{0}^{z} \frac{\bar{n}_{e}\left(z^{\prime}\right)}{1+z^{\prime}} \frac{d x}{d z^{\prime}} d z^{\prime}
$$

where $\bar{n}_{e}$ is the mean free-electron density,

$$
\bar{n}_{e}=\frac{\chi \rho_{\mathrm{g}}(z)}{\mu_{e} m_{p}}
$$

$\rho_{\mathrm{g}}(z)=\rho_{\mathrm{g}, 0}(1+z)^{3}$ is the mean gas density density of the Universe at redshift $z$, and $\mu_{e} m_{p}$ is the mean mass per electron, where $\mu_{e}=1.14$. We define $\chi$ as the fraction 
of the total number of electrons that are ionized. We assume that, for $z<z_{\text {rei }}$, hydrogen is completely ionized, so $\chi$ is dependent on the abundance and ionization state of helium,

$$
\chi=\frac{1-Y_{p}\left(1-N_{\mathrm{He}} / 4\right)}{1-Y_{p} / 2},
$$

where $Y_{p}$ is the primordial helium abundance and $N_{\mathrm{He}}$ the number of helium electrons ionized (which can be a function of redshift). Thus for $Y_{p}=0.24, \chi=\{0.86,0.93$, $1\}$ for neutral, singly, and fully ionized helium. For our fiducial model we assume $\chi=0.86$ (i.e., $N_{\mathrm{He}}=0$ ) at all redshifts. We explore the effect of helium reionization on the kSZ power spectrum in Section 5.

Writing $n_{e}=\bar{n}_{e}(1+\delta)$ we define the density weighted peculiar velocity $\mathbf{q}=\mathbf{v}(1+\delta)$, so that

$\frac{\Delta T}{T_{\mathrm{cmb}}}(\hat{\mathbf{n}})=\frac{\sigma_{T} \rho_{\mathrm{g}, 0}}{\mu_{e} m_{p} c} \int_{0}^{z_{\mathrm{rei}}} \frac{d x}{d z} d z(1+z)^{2} \chi \exp (-\tau(z)) \hat{\mathbf{n}} \cdot \mathbf{q} \cdot$

KSZ temperature fluctuations are generated by the projected contribution of ionized gas with a non-zero peculiar velocity along the line of sight. A key property of the $\mathrm{kSZ}$ signal is that Fourier modes of $\mathbf{q}(\tilde{\mathbf{q}}(\mathbf{k}))$ which have $\mathbf{k}$ parallel to $\hat{\mathbf{n}}$ suffer severe cancellation when projected along the line of sight. Therefore, only modes of $\tilde{\mathbf{q}}(\mathbf{k})$ parallel to $\hat{\mathbf{n}}$ but perpendicular to $\mathbf{k}$ can contribute (see Jaffe \& Kamionkowski 1998, for a rigorous demonstration of this. Henceforth we use ${ }^{\sim}$ to denote a Fourier space quantity.).

$\mathbf{q}$ can be decomposed into divergence free $\left(\mathbf{q}_{B}\right)$ and curl-free $\left(\mathbf{q}_{E}\right)$ components, which satisfy $\nabla \cdot \mathbf{q}_{B}=0$ and $\nabla \times \mathbf{q}_{E}=0$, respectively. In the Fourier domain, $\tilde{\mathbf{q}}=$ $\tilde{\mathbf{q}}_{E}+\tilde{\mathbf{q}}_{B}$, where $\tilde{\mathbf{q}}_{E}=\hat{\mathbf{k}}(\tilde{\mathbf{q}} \cdot \hat{\mathbf{k}})$ and

$$
\tilde{\mathbf{q}}_{B}(\mathbf{k})=\tilde{\mathbf{q}}-\hat{\mathbf{k}}(\tilde{\mathbf{q}} \cdot \hat{\mathbf{k}}) \text {. }
$$

When projected along the line of sight, the peaks and troughs of the component of $\tilde{\mathbf{q}}$ parallel to $\mathbf{k}, \tilde{\mathbf{q}}_{E}$, will cancel. Therefore, only the component of $\tilde{\mathbf{q}}$ perpendicular to $\mathbf{k}, \tilde{\mathbf{q}}_{B}$, contributes to the kSZ signal Vishniac 1987; Jaffe \& Kamionkowski 1998).

$\tilde{\mathbf{q}}_{B}$ can be written as a convolution between the Fourier transform of the velocity and density fields,

$$
\tilde{\mathbf{q}}_{B}(\mathbf{k})=\int \frac{d^{3} \mathbf{k}^{\prime}}{(2 \pi)^{3}}\left(\hat{\mathbf{k}}^{\prime}-\mu \hat{\mathbf{k}}\right) \tilde{v}\left(k^{\prime}\right) \delta_{b}\left(\left|\mathbf{k}-\mathbf{k}^{\prime}\right|\right),
$$

where $\mu=\hat{\mathbf{k}} \cdot \hat{\mathbf{k}}^{\prime}$. In the linear regime, $\tilde{\mathbf{v}}(\mathbf{k})$ is parallel to $\hat{\mathbf{k}}$, so only the $\mathbf{v} \delta$ component of $\mathbf{q}$ can contribute to $\mathbf{q}_{B}$. The $\mathrm{kSZ}$ power spectrum is thus generated by the crossterm $\delta \mathbf{v}$ (Ostriker \& Vishniac 1986; Vishniac 1987).

In the small angle limit, the kSZ angular power spectrum can be calculated using Limber's approximation,

$$
\begin{aligned}
C_{\ell}= & \frac{8 \pi^{2}}{(2 \ell+1)^{3}}\left(\frac{\sigma_{T} \rho_{\mathrm{g}, 0}}{\mu_{e} m_{p} c}\right)^{2} \int_{0}^{z_{r e i}}(1+z)^{4} \chi^{2} \Delta_{B}^{2}(\ell / x, z) \\
& \times \exp (-2 \tau(z)) x \frac{d x}{d z} d z
\end{aligned}
$$

where $k=\ell / x, \Delta_{B}^{2}(k, z)=k^{3} P_{B}(k, z) /\left(2 \pi^{2}\right)$, and the power spectrum of the curl component of the momentum field, $P_{B}$, is defined by $(2 \pi)^{3} P_{B} \delta\left(\mathbf{k}-\mathbf{k}^{\prime}\right)=$ $\left\langle\tilde{\mathbf{q}}_{B}(\mathbf{k}) \tilde{\mathbf{q}}_{B}^{*}\left(\mathbf{k}^{\prime}\right)\right\rangle$.
Vishniac (1987) first calculated an expression for $\Delta_{R}^{2}$ (see also Jaffe \& Kamionkowski 1998; Dodelson \& Jubas 1995; Ma \& Fry 2002),

$$
\begin{aligned}
\Delta_{B}^{2}(k)= & \frac{k^{3}}{2 \pi^{2}} \int \frac{d^{3} k^{\prime}}{(2 \pi)^{3}}\left[\left(1-\mu^{2}\right) P_{\delta \delta}\left(\left|\mathbf{k}-\mathbf{k}^{\prime}\right|\right) P_{v v}\left(k^{\prime}\right)\right. \\
& \left.-\frac{\left(1-\mu^{2}\right) k^{\prime}}{\left|\mathbf{k}-\mathbf{k}^{\prime}\right|} P_{\delta v}\left(\left|\mathbf{k}-\mathbf{k}^{\prime}\right|\right) P_{\delta v}\left(k^{\prime}\right)\right]
\end{aligned}
$$

where $P_{\delta \delta}$ and $P_{v v}$ are the linear theory density and velocity power spectra, and $P_{\delta v}$ is the density-velocity cross spectrum (for clarity we have suppressed the redshift dependence of $\Delta_{B}^{2}(k)$ and $\left.P(k)\right)$. In the linear regime, the continuity equation allows us to relate the peculiar velocity field with density perturbations, $\tilde{\mathbf{v}}(\mathbf{k})=$ $i \hat{\mathbf{k}}(f \dot{a} / k) \tilde{\delta}(\mathbf{k})$, where $f=d \log D / d \log a$ and $D$ is the linear growth factor. Therefore,

$$
P_{v v}(k)=\left(\frac{f \dot{a}}{k}\right)^{2} P_{\delta \delta}(k) ; \quad P_{\delta v}(k)=\left(\frac{f \dot{a}}{k}\right) P_{\delta \delta}(k) .
$$

Plugging these into Equation 9, we obtain

$\Delta_{B}^{2}(k)=\frac{k^{3}}{2 \pi^{2}} \dot{a}^{2} f^{2} \int \frac{d^{3} k^{\prime}}{(2 \pi)^{3}} P_{\delta \delta}\left(\left|\mathbf{k}-\mathbf{k}^{\prime}\right|\right) P_{\delta \delta}\left(k^{\prime}\right) I\left(k, k^{\prime}\right)$,

where

$$
I\left(k, k^{\prime}\right)=\frac{k\left(k-2 k^{\prime} \mu\right)\left(1-\mu^{2}\right)}{k^{\prime 2}\left(k^{2}+k^{\prime 2}-2 k k^{\prime} \mu\right)} .
$$

Combining Equations 8 and 9 gives the well-known Ostriker-Vishniac effect, which is the linear-theory part of the kinetic SZ power spectrum.

\subsection{Non-linear Contributions}

Several previous studies have investigated the impact of non-linear structure formation on the kSZ power spectrum, showing that non-linear corrections become large for angular scales $\ell>1000(\mathrm{Hu}$ 2000; Ma \& Frv 2002; Zhang et al. 2004). Hu (2000) and Ma \& Fry (2002) demonstrate that one can include the effect of non-linear density fluctuations to the kSZ power spectrum by exchanging the linear theory matter power spectrum, $P_{\delta \delta}$, in Equation 11 with the non-linear matter power spectrum, $P_{\delta \delta}^{\mathrm{NL}}$, so that

$\Delta_{B}^{2}(k)=\frac{k^{3}}{2 \pi^{2}} \dot{a}^{2} f^{2} \int \frac{d^{3} k^{\prime}}{(2 \pi)^{3}} P_{\delta \delta}^{\mathrm{NL}}\left(\left|\mathbf{k}-\mathbf{k}^{\prime}\right|\right) P_{\delta \delta}\left(k^{\prime}\right) I\left(k, k^{\prime}\right)$.

Ma \& Fry (2002) argue that the kinetic SZ signal is less sensitive to non-linear velocity fluctuations than non-linear density fluctuations due to the $1 / k^{2}$ weighting in the former. Using hydrodynamical simulations, Zhang et al. (2004); Shao et al. (2011) show that, for $k>2 h \mathrm{Mpc}^{-1}$, the power in the curl component of the velocity field (generated by non-linear gravitational collapse) can exceed the linear theory prediction. To account for this, they suggest an phenomenological correction in which $P_{\delta \delta}\left(k^{\prime}\right)$ is replaced by its non-linear counterpart. In this work, we follow the non-linear correction proposed by Ma \& Fry (2002) given in Equation 13. 
However, we investigate to what degree velocity fluctuations in the non-linear regime may effect the kSZ power spectrum in Section 5 ,

Throughout this work, we calculate the non-linear density power spectrum, $P_{\delta \delta}^{\mathrm{NL}}$ using the HALOFIT prescription of Smith et al. (2003). We find that the dark matter power spectrum predicted by HALOFIT is within $8 \%$ of that measured in our non-radiative simulations for $k<$ $4 \mathrm{~h} \mathrm{Mpc}^{-1}$ (Rudd et al. 2008), although it systematically underestimates the power spectrum at $k>1 h \mathrm{Mpc}^{-1}$. Heitmann et al. (2010) found similar results comparing HALOFIt to a large suite of N-body simulations over a wide range of cosmological models.

There is a much larger discrepancy between HALOFIT and our simulation that includes cooling and starformation (CSF). The formation of dense clumps of stars and gas at the center of halos produces a steepening of the dark matter density profile at small radii, increasing the density of the halo core. This process is sometimes referred to as 'halo (or adiabatic) contraction' (Blumenthal et al. 1986; Gnedin et al. 2004, 2011). The matter power spectrum measured in dark matteronly simulations, or by halo models calibrated on such simulations (such as HALOFIT), no longer matches that measured in simulations that include radiative cooling at wavenumbers $k>1 \mathrm{~h} \mathrm{Mpc}^{-1}$ (Jing et al. 2006; Rudd et al. 2008; Duffy et al. 2010; van Daalen et al. 2011).

We incorporate the effects of halo contraction using the simple modification to HALOFIT suggested by Rudd et al. (2008). This is implemented by multiplying the matter power spectrum by the ratio of Fouriertransformed NFW density profiles (Navarro et al. 1997) with two different concentrations,

$$
P_{\mathrm{DMcsf}}(k, z)=\Phi(k, z)^{2} P_{\text {DMonly }}(k, z),
$$

where

$$
\Phi(k, z)=\left[\frac{\lambda\left(R_{\mathrm{vir}} k / c_{2}, c_{2}\right)}{\lambda\left(R_{\mathrm{vir}} k / c_{1}, c_{1}\right)}\right] .
$$

$\lambda\left(R_{\mathrm{vir}}, k, c\right)$ is the Fourier transform of an NFW halo of virial radius $R_{\mathrm{vi}}$, 1 , and $\left(c_{2}, c_{1}\right)$ are the concentrations of the halo including and omitting the effects of radiative cooling and star-formation, respectively $\sqrt{5}$. We apply the correction to the matter power spectrum given by Equation [14 using $c_{1}=5, c_{2}=8.5$ and $R_{\mathrm{vir}}(z=0.55)=$ 1.1 comoving $\mathrm{Mpc} / h$ (corresponding to a virial mass of $\left.M_{\text {vir }}=1.07 \times 10^{14} h^{-1} M_{\odot}\right)$.

This procedure is clearly a very simplistic approximation to the effects of halo contraction on the matter power spectrum. The correction to the power spectrum is determined entirely by the effect of a change in concentration on the density profile of a halo with a characteristic mass and redshift. However, in Section 4 we demonstrate that it does provide a good agreement between our model and the CSF simulations at small scales.

\subsection{Thermal Pressure of Baryons}

4 The virial mass and radius are defined by $M_{\mathrm{vir}}=$ $\frac{4}{3} \pi R_{\mathrm{vir}}^{3} \Delta_{c} \rho_{c}(z)$, where $\Delta_{c}$ is the virial overdensity given in Bryan \& Norman (1998) and $\rho_{c}(z)$ is the critical density at redshift $z$

${ }^{5}$ See Rudd et al. (2008) for an analytic expression for $\lambda$.

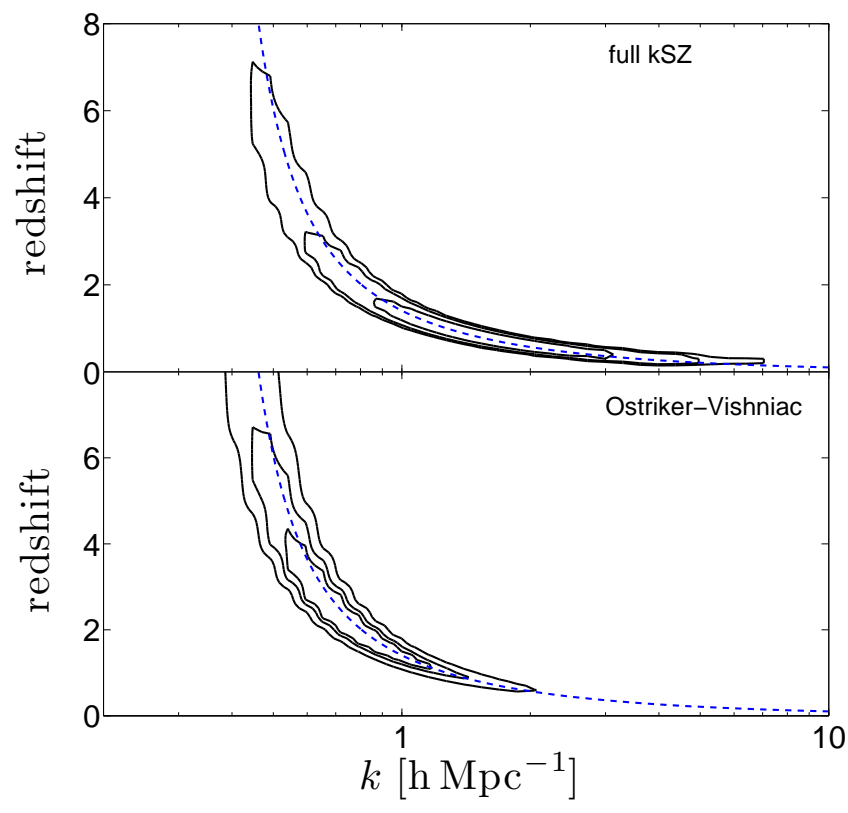

FIG. 1.- The contribution, $d^{2} C_{\ell} / d z d \ln (k)$, to the total kinetic SZ power at $\ell=3000$ by density fluctuations at a given redshift and spatial scale. In each panel, the contours enclose the regions that contribute 25, 50 and $75 \%$ of the total power (from inner to outer contours). The top panel shows mass and redshift dependence for the full kinetic SZ power spectrum including the non-linear density fluctuations. The lower panel shows the same contours for the Ostriker-Vishniac effect, in which only the linear density power spectrum is used. The dashed blue lines represent the size-distance relation, $k=\ell / x(z)$, for $\ell=3000$. Kinetic SZ power is generated by density fluctuations in the range $0.3 \leq k \leq 10 h \mathrm{Mpc}^{-1}$, with nonlinear fluctuations boosting the signal in the high- $k$, low redshift regime.

In previous work, it has commonly been assumed that $\delta_{\text {gas }}=\delta_{\mathrm{DM}}$, that is, perturbations in the gas density exactly follow those of the dark matter at all scales (Dodelson \& Jubas 1995; Jaffe \& Kamionkowski 1998; Ma \& Fry 2002; Zhang et al. 2004). However, thermal pressure between baryons should erase density fluctuations in the gas distribution at small scales. Furthermore, radiative cooling and star-formation can significantly modify the gas density power spectrum at both large and small scales (Jing et al.|2006; Rudd et al. 2008; Casarini et al. 2011; van Daalen et al. 2011).

In order to accurately predict the $\mathrm{kSZ}$ power spectrum we must be able to relate the power spectrum of gas density fluctuations, $P_{\text {gas }}$ (or more precisely, fluctuations in the number density of free electrons), with that of the dark matter. We incorporate the effects of baryon physics into a window function $W(k)$, such that

$$
P_{\text {gas }}^{\mathrm{NL}}(k, z)=W^{2}(k, z) P_{\mathrm{DM}}^{\mathrm{NL}}(k, z) .
$$

This then replaces the non-linear matter power spectrum in Equation 13. Note that we assume the velocity field of the gas follows that of the dark matter, which is a reasonable assumption at large scales.

In the non-radiative regime, the qualitative shape of $W^{2}(k)$ is simple to imagine. At large scales, and before the onset of gravitational collapse, we expect $W^{2}(k) \approx 1$. However, at small scales, the gas thermal pressure force suppresses gas density perturbations and so $W^{2}(k)$ will 
tend to zero as $k$ increases. Therefore, $W^{2}(k)$ acts as a filter, smoothing the gas density at some characteristic scale. As we shall demonstrate, the form of $W^{2}(k)$ for our simulation including radiative cooling and star-formation has a more complex dependence on $k$.

Gnedin \& Hui (1998) demonstrate that, for coupled density perturbations in the linear regime, the ratio of gas to dark matter density fluctuations is well described by the form

$W_{\mathrm{G} 98}(k, z)=0.5\left(\exp \left(-k^{2} / k_{f}^{2}\right)+\frac{1}{\left[1+4\left(k / k_{f}\right)^{2}\right]^{1 / 4}}\right)$,

where the (redshift-dependent) characteristic filter scale $k_{f}$ is given by

$$
\frac{1}{k_{f}^{2}(t)}=\frac{1}{D(t)} \int_{0}^{t} a^{2}\left(t^{\prime}\right) d t^{\prime} \frac{\ddot{D}\left(t^{\prime}\right)+2 H\left(t^{\prime}\right) \dot{D}\left(t^{\prime}\right)}{k_{J}^{2}\left(t^{\prime}\right)} \int_{t^{\prime}}^{t} \frac{d t^{\prime \prime}}{a^{2}\left(t^{\prime \prime}\right)} .
$$

Here $k_{\mathrm{J}}$ is the Jean's scale,

$$
k_{\mathrm{J}}(t)=\frac{a}{c_{\mathrm{S}}(t)} \sqrt{4 \pi G \rho_{m}(t)},
$$

$\rho_{m}$ is the mean matter density of the Universe and $c_{\mathrm{S}}=$ $\sqrt{d P / d \rho}$ is the mean sound speed at time $t$. As noted by Gnedin et al. (2003), the filter scale at a given time is not directly proportional to the Jeans scale at the same time, but to the integral over the thermal history of the gas up to that point.

$\mathrm{Hu}$ (2000) and McQuinn et al. (2005) used Eq. 17 to approximate the effects of thermal pressure in their calculations of the (post-reionization) $\mathrm{kSZ}$ power spectrum. In this work, we measure the shape and evolution of $W^{2}(k)$ in hydrodynamic simulations and apply the results to our model for the power spectrum.

\subsection{Final Model}

Our full model for the $\mathrm{kSZ}$ power spectrum is given by Equation 8 , where the expression for $\Delta_{B}^{2}$ including non-linear corrections, the gas window function and halo contraction is

$$
\begin{aligned}
\Delta_{B}^{2}(k)= & \frac{k^{3}}{2 \pi^{2}} \dot{a}^{2} f^{2} \int \frac{d^{3} k^{\prime}}{(2 \pi)^{3}} W^{2}\left(\left|\mathbf{k}-\mathbf{k}^{\prime}\right|\right) \Phi^{2}\left(\left|\mathbf{k}-\mathbf{k}^{\prime}\right|\right) \\
& \times P_{\delta \delta}^{\mathrm{NL}}\left(\left|\mathbf{k}-\mathbf{k}^{\prime}\right|\right) P_{\delta \delta}\left(k^{\prime}\right) I\left(k, k^{\prime}\right)
\end{aligned}
$$

We remind the reader that the superscript NL represents the non-linear matter power spectrum, calculated using the HaloFit procedure of Smith et al. (2003). Otherwise, we use the linear-theory matter power spectrum.

One of the principal aims of this work is to explore the impact of astrophysical processes such as radiative cooling, star-formation and supernova feedback on the $\mathrm{kSZ}$ power spectrum. This is achieved by measuring the window function $W^{2}(k)$ in hydrodynamic simulations both including and omitting these processes. By plugging the results into our model, we are able to calculate the effect of these processes on the kSZ power spectrum. To investigate the impact of non-linear structure formation and halo contraction, we measure the curl component of the momentum power spectrum, $\Delta_{B}^{2}$, directly from our simulations and compare with the analytic prediction given by Equation 20
Based on our hydrodynamic simulations, we investigate three models for the kinetic SZ power spectrum, labeled DM (dark matter), NR (non-radiative) and CSF (cooling and star-formation). The three models differ principally in the gas window function, $W_{i}^{2}(k)$, used to relate the non-linear dark matter power spectrum to the gas density power spectrum, where $i=\left\{1, W_{\mathrm{NR}}^{2}, W_{\mathrm{CSF}}^{2}\right\}$ for the $\{\mathrm{DM}, \mathrm{NR}, \mathrm{CSF}\}$ models. For the CSF model we also include the halo contraction correction to the dark matter density power spectrum, $\Phi(k, z)$, given by Equation 14. For the DM and NR models we set this equal to 1 at all $k$ and $z$.

In our model, simulations are used to determine the gas density power spectrum whereas the velocity modes are entirely calculated from linear theory. Our technique thus circumvents the problems relating to the truncation of large-scale velocity modes due to a limited simulation box size (see Section 4.2). Indeed, our simulations accurately resolve the range of spatial scales over which density fluctuations contribute significantly to the kinetic SZ power spectrum for $1000 \leq \ell \leq 10000$. We now demonstrate this point explicitly.

In Figure 1 we plot the contribution to the total kinetic SZ power at $\ell=3000$ by density fluctuations at a given redshift and spatial scale $k=3000 / x$. In each panel, the contours enclose the regions that contribute 25, 50 and $75 \%$ of the total power (inner to outer contours). The top panel shows contours for the full kinetic SZ power spectrum including the non-linear density fluctuations. The lower panel shows the same contours for the OstrikerVishniac effect, in which only the linear-regime matter power spectrum is used. The dashed blue lines show the relation $k=\ell / x(z)$ at $\ell=3000$. The shape of the contours follow that expected for the distance-size relation at a constant angular scale. In this figure $W^{2}(k)$ is fixed to 1 at all scales.

Comparing the two panels clearly demonstrates the impact of non-linear density perturbations on the $\mathrm{kSZ}$ power. The full $\mathrm{kSZ}$ contours extend to larger $k$ and lower redshift, where the impact of the non-linear corrections to the power spectrum are largest. More than $50 \%$ of the full $\mathrm{kSZ}$ power is sourced by density fluctuations in the range $0.6 \leq k \leq 5 \mathrm{hMpc}^{-1}$ and $0 \leq z \leq 3$, while wavenumbers up to $k=8 \mathrm{hpc}^{-1}$ provide a non-negligible contribution. In contrast, we find that the principal contribution of velocity modes to the $\mathrm{kSZ}$ signal comes from the range of scales encompassed by $0.005 \leq k \leq 0.5 h \mathrm{Mpc}^{-1}$ and thus from much smaller $k$ than the density fluctuations. This emphasizes that the kinetic SZ power spectrum is generated by small scale density fluctuations caught up in large scale velocity flows. The OV signal is spread over a larger redshift range, but limited to contributions from density fluctuations at $k \leq 2 h \mathrm{Mpc}^{-1}$. Our simulations accurately resolve density fluctuations over the full range of $k$ plotted and are thus well-suited to calibrating our model.

\section{SIMULATIONS}

Our simulations are performed using the Adaptive Refinement Tree (ART) N-body+gas-dynamics code (Kravtsov et al. 2002; Rudd et al. 2008), which is an Eulerian code that uses adaptive refinement in space and time, and non-adaptive refinement in mass (Klypin et al. 
TABLE 1

List of Simulations

\begin{tabular}{lcccccccccc}
\hline \hline Name & $\begin{array}{c}L_{\mathrm{box}} \\
\left(h^{-1} \mathrm{Mpc}\right)\end{array}$ & $\Omega_{m}$ & $\Omega_{\Lambda}$ & $h$ & $\Omega_{b} h^{2}$ & $\sigma_{8}$ & $n_{s}$ & $n_{D M}$ & $\begin{array}{c}m_{p} \\
\left(10^{9} h^{-1} M_{\odot}\right)\end{array}$ \\
\hline L60NR & 60 & 0.3 & 0.7 & 0.7 & 0.021 & 0.9 & 1.0 & $256^{3}$ & 0.92 \\
L60CSF & 60 & 0.3 & 0.7 & 0.7 & 0.021 & 0.9 & 1.0 & $256^{3}$ & 0.92 \\
BolshoiNR & 250 & 0.27 & 0.73 & 0.7 & 0.023 & 0.82 & 0.95 & $1024^{3}$ & 1.08 \\
\hline
\end{tabular}

Note. $-L_{\text {box }}$ is the simulation box side-length, $n_{D M}$ and $m_{p}$ are the number of dark of dark matter particles and their mass (in units of $\left.10^{9} h^{-1} M_{\odot}\right)$, and $\Delta x$ is the peak spatial resolution of the simulation.

2001) to achieve the dynamic range necessary to resolve the cores of halos formed in self-consistent cosmological simulations.

The simulation properties, their associated box sizes, cosmological parameters, and resolutions are summarized in Table 1. Two of the three simulations (BolshoiNR and L60NR) are performed in the non-radiative regime where baryons are shock heated during structure formation but unable to radiatively cool. BolshoiNR is a large simulation designed to study the formation of galaxy clusters. L60NR and L60CSF are realizations of the same set of initial density fluctuations simulated with varying physical processes included and were previously used to study baryon effects on the matter power spectrum in Rudd et al. (2008).

A complete description of the physical processes implemented in the L60CSF simulation can be found in Rudd et al. (2008). Briefly, gas is converted to collisionless stellar particles using a Kennicutt-Schmidt type density relation for a gas consumption timescale of $\sim$ 2.7Gyr. Mass, energy, and metals are returned to the ISM through prescriptions for types II and Ia supernovae and stellar mass-loss via winds. Radiative cooling rates are tabulated over a range of gas temperature, density, and metallicity including a redshift-dependent cosmological UV background (Haardt \& Madau 1996) using the CLOUDY code (ver. 96b4; Ferland et al. 1998). In constructing these rates the collisional and UV ionization equilibria are explicitly calculated. This allows the direct determination of the fraction of ionized electrons in each mesh cell, $\chi$, rather than assuming a universally averaged value.

One of the main effects of cooling and star formation is to lower the volume-averaged gas density significantly below the cosmic baryon density due to the conversion of gas into stars. At $z=4$ the gas fraction is $\rho_{\mathrm{g}} / \rho_{\text {bar }}=0.99$ (where $\rho_{\text {bar }}$ is the mean baryon density of the Universe), whereas by $z=0$ it has fallen to 0.8. As noted by Rudd et al. (2008), the conversion of gas into stars in the simulation is significantly more efficient than is observed - especially in the highest density regions - due to the well-known over-cooling problem (see also Kravtsov et al. 2005, 2009). At the high-mass end, the halos in this simulation have a stellar mass fraction at $z=0$ that is approximately $50 \%$ greater than observed for group and cluster-mass objects (Gonzalez et al. 2007; Giodini et al. 2009). We note that, while the star-formation rate at high redshift $z>4$ is likely to be underestimated (as many halos are poorly resolved at these epochs, Springel \& Hernquist 2003), Figure 1 indicates that the majority of the $\mathrm{kSZ}$ power at $\ell=3000$ is sourced at lower redshift. Overall, the
L60CSF simulations should therefore underestimate the amplitude of the gas density - and thus the kinetic SZ power spectrum. On the other hand, the absence of these processes in our non-radiative simulations results in a mean gas density in halos that is substantially larger than that observed. The BolshoiNR simulation should thus over-estimate the kinetic SZ power. Therefore, while neither simulation represents the real Universe, we expect them to provide well-motivated lower and upper limits to theoretical estimates of the kSZ power spectrum. These limits can be utilized to place constraints on the $\mathrm{kSZ}$ signal from reionization.

To compare with these two limiting cases we also reran the L60CSF simulation having turned off radiative cooling at $z=1.8$ (henceforth referred to as L60CSFz2). While this run should be considered unrealistic, it serves two useful purposes. Firstly, it allows us to investigate whether a radical change in the star-formation prescription can significantly modify the shape of the window function, $W^{2}(k)$, and the $\mathrm{kSZ}$ power spectrum away from that predicted by either the BolshoiNR or L60CSF simulations. This then enables us to evaluate how robust our estimate of $W^{2}(k)$ is to changes in baryon physics: does it vary smoothly between the non-radiative and overcooling case, or does it depend sensitively on the starformation prescription? Secondly, the final stellar (and gas) mass fraction in halos of mass $M_{500}>10^{12} h^{-1} M_{\odot}$ at $z=0$ in this simulation lies between the values produced by the BolshoiNR and L60CSF simulations. For example, for halos of mass $M_{500}=5 \times 10^{13} \mathrm{M}_{\odot}$, the ratio of stellar (gas) mass to total mass in the L60CSFz2 simulation is approximately $0.12(0.8)$ of the universal baryon fraction, compared to 0 (0.9) for BolshoiNR and 0.6 (0.45) for L60CSF. Therefore, this run provides a useful intermediary case between the full cooling plus star-formation and non-radiative cases. In Section 6 we discuss future work that is required to make precision estimates of the kinetic SZ power spectrum.

The BolshoiNR and L60CSF are the primary simulations used to gauge the effects of cooling and star formation and to calibrate our models. As indicated in Table1. the BolshoiNR simulation has the largest box size (250 $\mathrm{Mpc} / h)$. The range of scales resolved by this simulation encompasses the wavenumbers $0.03 \leq k \leq 100 h \mathrm{Mpc}^{-1}$ (we conservatively use an upper limit of $1 / 8$ the Nyquist wavenumber corresponding to the spatial resolution). Mildly overdense structures $(\delta \approx 10)$ are followed at a grid resolution of approximately $60 h \mathrm{kpc}^{-1}$, corresponding to an upper limit on $k$ of about $7 \quad h \mathrm{Mpc}^{-1}$. Therefore, this simulation has adequate resolution in low density regions to account for the potential con- 
tribution of filamentary structures to the kSZ signal (Atrio-Barandela et al. 2008). The L60CSF simulation has a box size of $60 \mathrm{Mpc} / \mathrm{h}$, resolving fluctuations over the range $0.1 \leq k \leq 110 h \mathrm{Mpc}^{-1}$. The resolution in lower-density regions is approximately $200 h \mathrm{kpc}^{-1}$, so it is possible that density fluctuations in these regions are slightly underestimated. However, Hallman et al. (2009) demonstrate that the contribution of regions of density $\delta<50$ to the $\mathrm{kSZ}$ power spectrum in their simulations is an order of magnitude below that of denser regions. Therefore, comparing with the upper panel of Figure 1. it is clear that our simulations adequately resolve density fluctuations over the range of scales that contribute the bulk of the kSZ signal.

While we do not directly present results from the L60NR simulation, we use this to check the effect of varying the simulation box size (by comparing BolshoiNR and L60NR) and separate it from the effect of adding baryonic physics (by comparing L60NR and L60CSF).

\section{RESULTS}

\subsection{Gas Window Function}

In the left panel of Figure 2 we plot the window function $W^{2}(k)=P_{\text {gas }}(k) / P_{\mathrm{DM}}(k)$ for the BolshoiNR simulation, where $P_{\text {gas }}$ and $P_{\mathrm{DM}}$ are the measured gas and dark matter density power spectra. The points show the results over a range of timesteps, corresponding to $a=0.4$ to 1.0 in steps of 0.1 (from upper to lower at $\left.k=2 h \mathrm{Mpc}^{-1}\right)$. The black solid lines are fits to the simulation results using the fitting function given below. The red lines show the linear-theory window function of Gnedin \& Hui (1998) given by Equation 17.

Fluctuations in the gas density follow those of the dark matter at large scales $\left(k<1 h \mathrm{Mpc}^{-1}\right)$, but are rapidly suppressed towards smaller scales as the gas thermal pressure begins to counter gravity in overdense regions. Note that the largest value of $k$ plotted is significantly below the maximum that is resolvable for the simulation. Hence, the truncation of power at small scales in $P_{\text {gas }}$ is due to a physical smoothing of the gas density by thermal interactions rather than an artificial smoothing due to the finite resolution of the simulation.

We find that the following fitting-function provides a good description of the BolshoiNR simulation results,

$$
W_{\mathrm{NR}}(k, a)=0.5\left(\exp \left(-k / k_{f}^{\prime}\right)+\frac{1}{1+\left(g(a) k / k_{f}^{\prime}\right)^{7 / 2}}\right),
$$

where $k_{f}^{\prime}=12.6 a^{-1}+6.3$ and $g(a)=0.84 a^{-1}$. We have verified that Equation 21 also provides a good match to the window function measured from the L60NR simulation. The difference between the BolshoiNR and L60NR simulation box sizes and cosmological parameters do not significantly affect our results.

At the final output, the characteristic filter scale, $k_{f}^{\prime}$, is approximately a factor of 3.3 less than that of the lineartheory prediction given by Equation [18. This is also evident from comparing the window function suggested by Gnedin \& Hui (1998, red lines) with the simulation results; the smoothing of the gas density fluctuations occurs at much smaller scales than in the simulation. The difference between the Gnedin \& Hui (1998) win- dow function and the simulation results is due to shock heating of the gas in the simulation as density perturbations become non-linear. To calculate the Jean's scale in Equation 18 we assume that the gas is initially coupled to the CMB temperature, but then evolves adiabatically, $T_{\text {gas }} \propto 1 / a^{2}$, until it reaches a minimum temperature of $300 \mathrm{~K}$ (which is also imposed in the simulation). The lower gas temperature at late times results in a large Jeans wavenumber, and thus the smoothing of the gas density is limited to very small scales (e.g., $\left\{k_{J}, k_{f}\right\} \approx\{32,63\} h \mathrm{Mpc}^{-1}$ at $\left.a=1\right)$. However, in the simulation, as halos collapse and grow, accretion shocks heat much of the gas to temperatures significantly above the $300 \mathrm{~K}$ temperature floor (note that this simulation, unlike the L60CSF run, does not include a UV background). The mean (mass-weighted) gas temperature in the simulation is in excess of $10^{6} \mathrm{~K}$ at $z=0$. Thus we see smoothing of the gas distribution at much larger scales in the simulation than predicted by purely adiabatic evolution.

On the right side of Figure 2 we show the window function, $W_{\mathrm{CSF}}(k)$, measured in our L60CSF simulation at the timesteps corresponding to $a=0.3$ to 1.0 in steps of 0.1 (black lines, from top to bottom at $k=1 \mathrm{~h} \mathrm{Mpc}^{-1}$; for clarity we represent the simulation results with lines rather than symbols). In this simulation we are able to measure the ionization fraction of hydrogen in each cell. We therefore plot the ratio of the free-electron (rather than the gas) density power spectrum to that of the dark matter. However, since the global neutral fraction is very small, using the gas density produces similar results.

The inclusion of gas cooling and star-formation produces a very different window function to the nonradiative simulation. Firstly, the overall amplitude at large scales is clearly time-dependent. At $k=$ $0.2 \mathrm{~h} \mathrm{Mpc}^{-1}, W^{2}(k)$ varies from 0.92 at $a=0.2$ to 0.62 at $a=1.0$. For the BolshoiNR simulation we found that $W_{\mathrm{NR}}^{2}(k=0.2) \approx 1$ at every timestep. The difference is due to the evolving gas fraction within halos in the CSF simulation. At $a=1$, the square of the ratio of the gas to total baryon density in the box is $\left(\rho_{\mathrm{g}} / \rho_{\mathrm{bar}}\right)^{2}=0.64$ (shown as the horizontal dot-dashed line). The low gas density within halos in this simulation significantly reduces the power spectrum of gas density fluctuations relative to the dark matter. This is particularly evident at low $k$ as the two-halo contribution to the gas density power spectrum is proportional to the square of the mean gas density in halos (Semboloni et al. 2011). Therefore, as the gas density is reduced, so is the amplitude of $W_{\mathrm{CSF}}^{2}(k)$ at large scales.

At intermediate scales $W^{2}(k)$ slowly decreases, but starts rising again at $k>5 h \mathrm{Mpc}^{-1}$. The increase in power towards small scales is due to the cooling of gas at the center of halos, steepening the density profile at small radii relative to the dark matter and thus boosting the small-scale power. For earlier outputs (upper lines) we see that the power in the free-electron density can greatly exceed that of the dark matter $\left(W_{\text {CSF }}^{2}(k)>1\right)$. With time, this bias gradually disappears due to two effects. Firstly, as the simulation progresses, the cold gas in halo cores is converted to stars. Secondly, the gas in the very center of halos can cool to the point at which hydrogen is no longer ionized, reducing the free-electron 

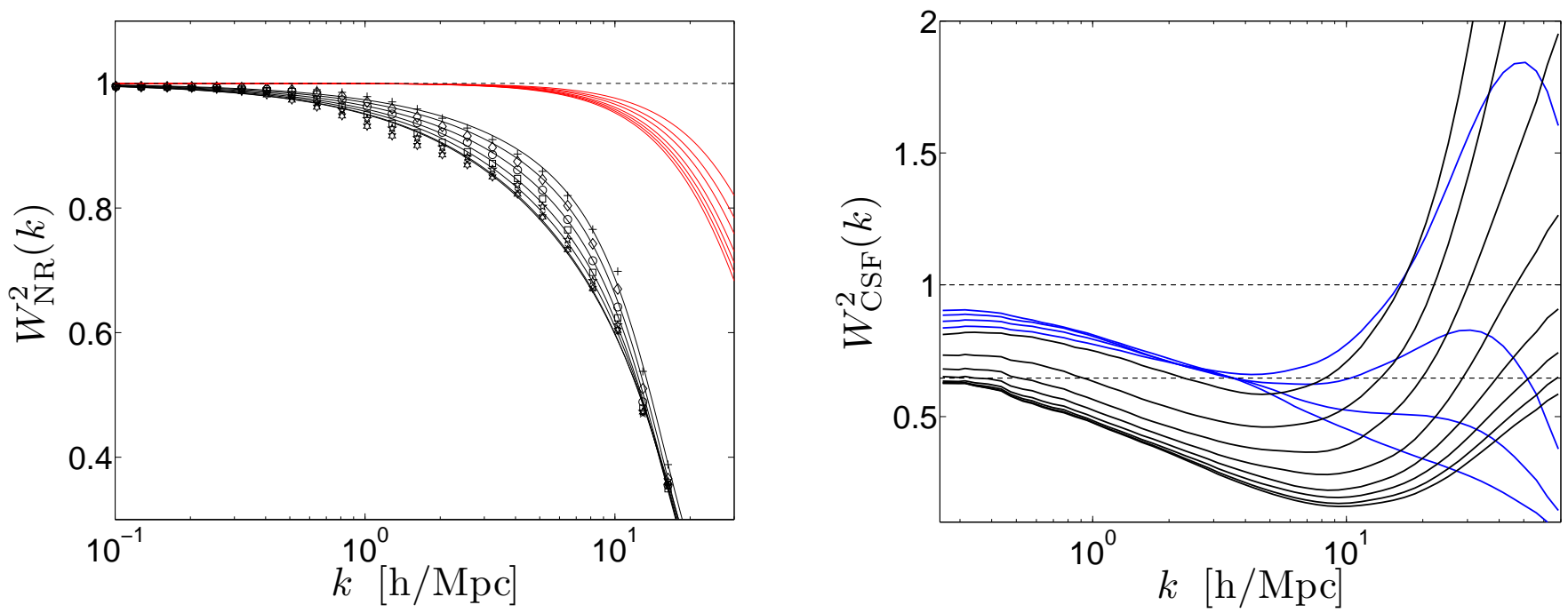

FIG. 2.- (left) The ratio of the gas density to dark matter density power spectra, $W^{2}(k)$, measured in the BolshoiNR simulation (black symbols) and the linear perturbation theory prediction of Gnedin \& Huil (1998) (red solid lines). For each, the results are shown from $a=0.4$ to 1 (where $a$ is the scale factor for the size of the universe) in steps of $\Delta a=0.1$ (from top to bottom at $k=2 h \mathrm{Mpc}^{-1}$ ). The black solid lines show the fit to the simulation points given by Equation 21 (right) The ratio of the free electron density to dark matter density power spectra in the L60CSF simulation (black lines). The results are shown from $a=0.3$ to 1 in steps of $\Delta a=0.1$ (from top to bottom). Also plotted is the ratio of the free electron density to dark matter density power spectrum for the L60CSFz2 simulation (in which radiative cooling is turned off at $z=1.8$ ) at $a=0.4,0.5,0.68$ and 1 (from top to bottom at $k=10 h \mathrm{Mpc}^{-1}$ )

density. However, the neutral fraction always remains small and thus the former is the dominant effect. Using the gas (rather than free-electron) power spectrum produces very similar results.

The blue lines in the right-hand panel of Figure 2 show the window function obtained for the L60CSFz2 simulation, in which radiative cooling is artificially turned off at $z_{\text {off }}=1.8$. The results are given for the timesteps corresponding to $a=0.4,0.5,0.68$ and 1 (from top to bottom at $k=10 h \mathrm{Mpc}^{-1}$ ). Switching off radiative cooling has a clear effect on the gas distribution across the range of scales probed. After $z_{\text {off }}$, the cold, dense clumps of gas in halo cores begin to heat and expand, rapidly suppressing small-scale density fluctuations. The reduction of smallscale power thus occurs in both CSF simulations, but for very different reasons: in L60CSF cold gas is converted into stars, whereas in L60CSFz2 the gas mixes with its warmer surroundings and begins to expand. This also results in a different behavior at large spatial scales. In L60CSFz2, the gas becomes less centrally concentrated in halos, reducing the suppression in power (relative to the dark matter) that is seen in the L60CSF simulation. As the simulation progresses towards $z=0, W^{2}(k)$ thus increases slightly at small $k$. By the final output, the shape of the window function is beginning to resemble that of the BolshoiNR simulation (left-hand panel of Figure 2). Therefore, once cooling is turned off, the window function obtained from the L60CSFz2 simulation begins to transition from a CSF-like shape to a NR-like shape. We show the effect of this on the kSZ power spectrum in Section 5.

The form of $W_{\mathrm{CSF}}^{2}(k)$ is evidently more complicated than $W_{\mathrm{NR}}^{2}(k)$. To incorporate the CSF window functions into our calculation of the kinetic SZ power spectrum, we instead interpolate between the simulation results for the redshifts between outputs. We assume that $W^{2}(k)$ remains fixed at its value at $k=0.2 h \mathrm{Mpc}^{-1}$ for scales with $k<0.2 h \mathrm{Mpc}^{-1}$. For epochs earlier than $a=0.3$ $(z>2.33)$, we assume that $W_{\mathrm{CSF}}^{2}(k)$ smoothly converges towards unity at all scales.

\subsection{Momentum Power Spectrum}

We now compare the power spectrum of the curl component of the gas momentum field, $\Delta_{B}^{2}$, predicted by our model with that measured directly from the simulations. This comparison enables us to test whether our model accurately captures the impact of non-linear structure growth and baryonic physics in the simulations. This is of particularly importance as the $\mathrm{kSZ}$ power spectrum is a weighted integral of $\Delta_{B}^{2}$ over redshift. We also use our model to demonstrate the impact on $\Delta_{B}^{2}$ of the truncation of large-scale velocity modes due to the finite size of our simulated volumes.

To measure $\Delta_{B}^{2}$ in our simulations we use a $1024^{3}$ mesh rather than the fully refined mesh in each simulation. Hence, we are limited to a narrower range of scales than for the density power spectra. For the BolshoiNR simulation (which has a box side-length of $250 \mathrm{Mpc} / h$ ) this range is $0.03 \leq k \leq 12 \mathrm{~h} \mathrm{Mpc}^{-1}$. For the L60CSF simulation (60 Mpc/ $/ h$ box) the range is $0.1 \leq k \leq 50$.

In Figure 3 we plot $\Delta_{B}^{2}$ for the BolshoiNR simulation at four timesteps, corresponding to $z=4,1.87,1$ and 0 . The momentum power is shown in terms of the dimensionless quantity $\Delta_{B} k / H(z)$. The black dots represent the measured simulation power spectrum. The blue lines give the analytic prediction in two regimes: the linear regime calculation (the Ostriker-Vishniac effect) given by Equation 11 (dotted), and our fiducial model given in Equation 20, which includes the non-linear corrections to the density power spectrum (solid). Both include the window function, $W_{\mathrm{NR}}^{2}(k)$.

Non-linear density fluctuations become significant when $\Delta_{b} k / H(z) \approx 1$. Our full non-linear model re- 


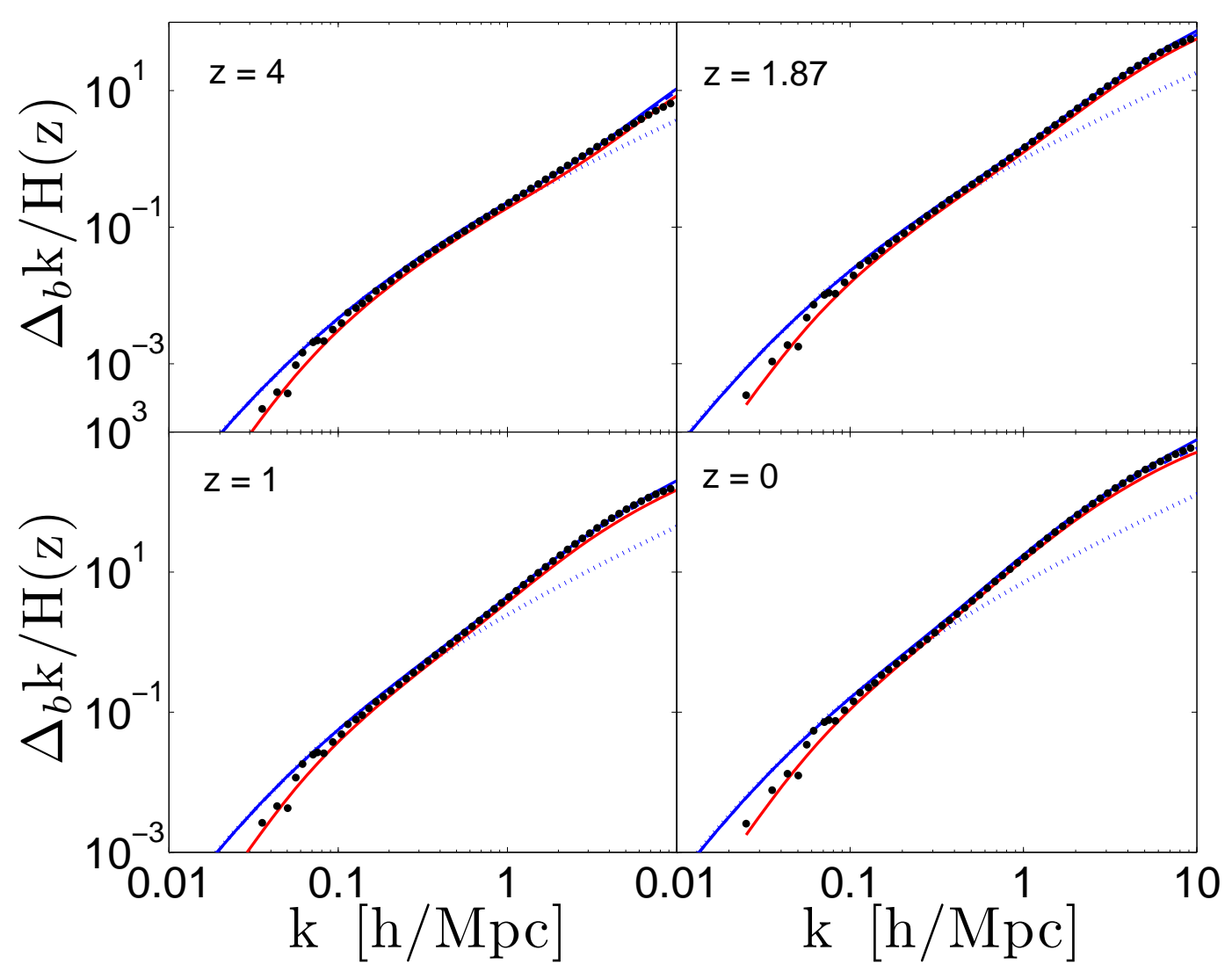

FIG. 3. - The power spectrum of the curl component of the momentum field in the BolshoiNR simulation at four timesteps: $z=4$, 1.87, 1 and 0 (top left, top right, lower left, lower right). The black dots represent the simulation results, the blue lines give the analytic prediction of $\Delta_{B} k / H(z)$ in two regimes: the linear regime calculation given by Equation 11] (dotted), and the non-linear version given by Equation 13 (solid). The red line shows the 'truncated' model (see text).

produces the simulation results extremely well at $k>$ $0.1 \mathrm{~h} \mathrm{Mpc}^{-1}$, but lies systematically above the simulation points at lower $k$. This is simply due to the finite volume of the simulation box. $\Delta_{B}^{2}(k)$ is generated by a convolution between velocity modes at wavenumber $k^{\prime}$ with density modes at $\left|k-k^{\prime}\right|$. The largest contribution comes from velocity modes at large scales coupling to density modes at smaller scales. Velocity modes on scales larger than the simulation box size are not accounted for, resulting in an underestimate of the momentum power.

To demonstrate that this is indeed the case, we recalculate $\Delta_{B}^{2}$, placing an upper and lower limit on the integral over $k^{\prime}$ in Equation 20 such that only the velocity modes encompassed by the simulation box are included. The red lines in each panel of Figure 3 show the results of this 'truncated' model. It accurately reproduces the simulation on all scales. This was previously demonstrated by Zhang et al. (2004), who performed a similar test by calculating a discretized version of Equation 20 and compared the results with their simulations. Despite the overall very good agreement, the truncated model does slightly underestimate the simulation results at $k>2 h \mathrm{Mpc}^{-1}$. This may be due to the impact of a non-linear, curl component, of the velocity field generated by shell-crossing during halo formation (as suggested by Zhang et al. 2004; Shao et al. 2011). To mitigate this, Zhang et al. (2004) suggest replacing $P_{\delta \delta}$ in
Equation 13 with it's non-linear counterpart. When we do so, we find that the ratio of model to simulation increases slightly, from 0.88 to 0.94 at $k=2 h \mathrm{Mpc}^{-1}$.

In Figure 4 we plot the curl component of the gas momentum power spectrum in the L60CSF simulation (black dots) at the same four timesteps. In this figure, the blue lines show the results omitting (dashed) and including (solid) the halo contraction correction applied to the (non-linear) density power spectrum described in Section 2.2. Both lines include the window function $W_{\mathrm{CSF}}^{2}(k)$ measured in the L60CSF simulation.

At the low- $k$ end, the simulation points again lie systematically below the model. However, for this simulation the discrepancy extends to much higher $k$. The L60CSF simulation box is more than a factor of four times smaller than the BolshoiNR simulation (see Table 11), and so the velocity mode truncation is more severe. We note that our L60NR simulation underestimates $\Delta_{b}^{2}$ (relative to our non-radiative model) by a similar amount, confirming that the box-size effect is independent of the baryonic physics included in the simulation. As in Figure 3, the red lines show our model prediction having limited the calculation to include only those scales encompassed by the simulation. The model again reproduces the simulation results very well (although at $a=1$ it appears to slightly overestimate the momentum power at scales around $k=1 h \mathrm{Mpc}^{-1}$ ). 


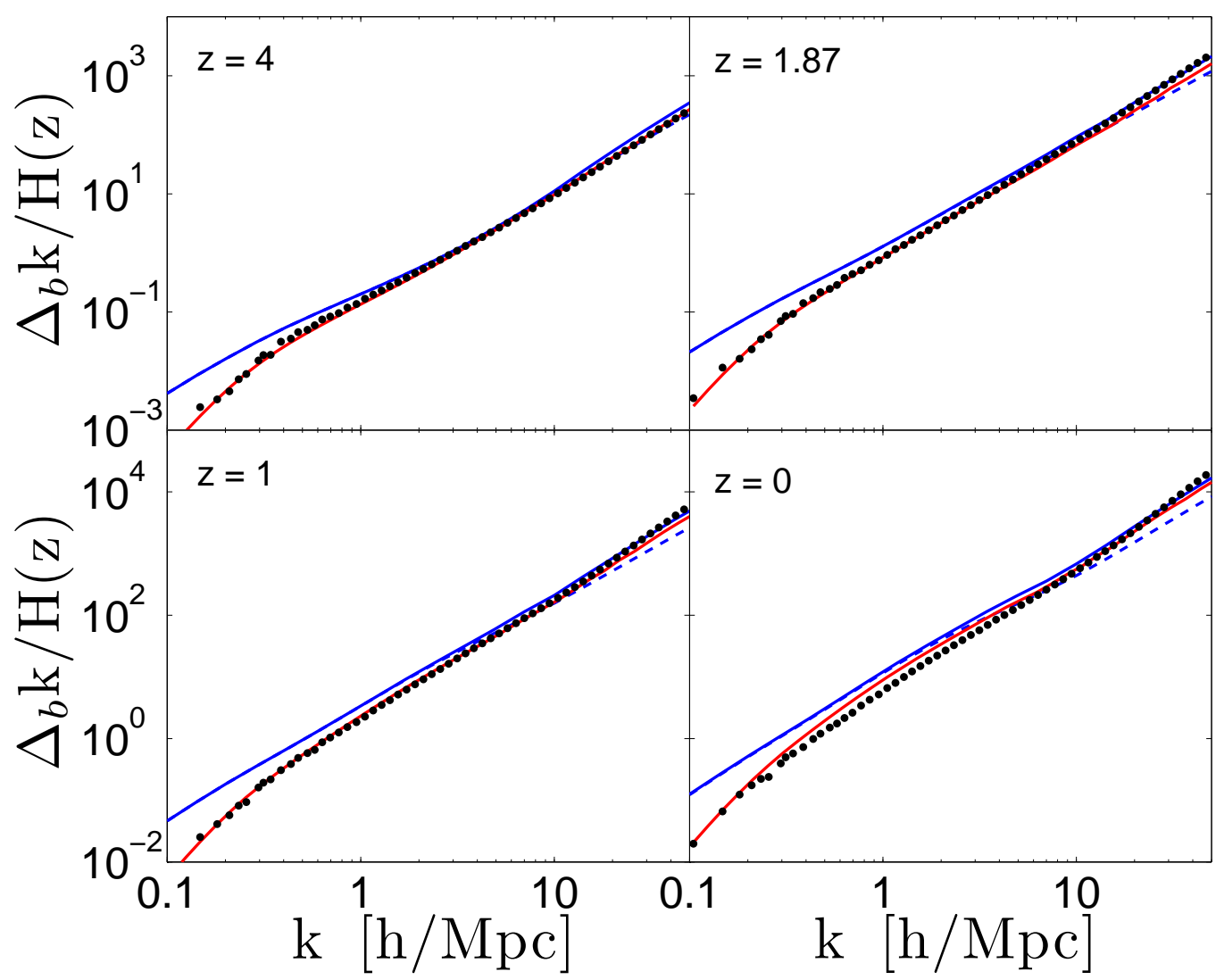

FIG. 4.- Similar to Figure 3 but for the L60CSF simulation. The black dots represent the simulation results, the solid and dashed blue lines give the analytic prediction of $\Delta_{B} k / H(z)$ including and omitting the halo contraction correction, respectively. The red line shows the 'truncated' model (see text).

The dashed lines demonstrate the model with no halo contraction correction. It is clear that, without this correction, the model underestimates $\Delta_{B}^{2}$ for wavenumbers $k>10 h \mathrm{Mpc}^{-1}$, especially at $z=0$. The solid blue line demonstrates that our simple correction does a reasonable job of reproducing the simulated momentum power spectrum. However, as Figure 1 demonstrates, these scales do not contribute significantly to the kSZ power spectrum at $\ell=3000$.

\section{KINETIC SZ POWER SPECTRUM}

We now utilize the gas window functions measured in our NR and CSF simulations to explore the impact of baryonic physics on the shape and amplitude of the kinetic SZ power spectrum. As described in Section 2.4, we evaluate three baseline models: DM, NR and CSF. For the DM matter model $W^{2}(k)=1$, for the $\mathrm{NR}$ and CSF we use the window functions $W_{\mathrm{NR}, \mathrm{CSF}}^{2}(k)$ measured in our simulations. We also investigate the redshift dependence of the $\mathrm{kSZ}$ power spectrum, and its scaling with cosmological parameters for each model. Finally we compare the results presented in this work with those of previous theoretical studies as well as the latest observational constraints.

\subsection{The Impact of Baryon Physics}

In Figure 5 we plot the kinetic SZ power spectrum for our three models: DM, NR and CSF (solid black, red and blue lines). The dashed lines represent variants to the DM and CSF models. The black dashed line shows the linear theory version of the DM model, which we label OV (Ostriker-Vishniac). The blue dashed line shows the kSZ power spectrum calculated using the L60CSFz2 simulation window function. The power spectra are plotted in terms of $D_{\ell}=\ell(\ell+1) C_{\ell} / 2 \pi$.

Comparing the dashed and solid black lines demonstrates the impact of non-linear structure growth on the kinetic SZ power spectrum. Including the non-linear corrections significantly boosts $\mathrm{kSZ}$ power at all but the largest angular scales. At $\ell=3000$, non-linear corrections have increased the $\mathrm{kSZ}$ signal by a factor of 2 . By $\ell=10,000$, this has increased to a factor of 3.5. The $\mathrm{kSZ}$ effect is sourced by small-scale density fluctuations caught up in large-scale bulk velocity flows. Therefore, the large boost in the amplitude of small-scale density perturbations due to non-linear gravitational collapse also enhances the kSZ power, especially at small angular scales.

The difference between the DM and NR models are small. At $\ell=3000(10,000)$ the BolshoiNR window function reduces the $\mathrm{kSZ}$ power from the DM model by 0.20 $(0.72) \mu \mathrm{K}^{2}$. The NR window function suppresses power at scales $k \gtrsim 8 \mathrm{~h} \mathrm{Mpc}^{-1}$ (see the left panel of Figure 2). As demonstrated by Figure 1, gas density fluctuations at these scales contribute little to the $\mathrm{kSZ}$ power spectrum (at $\ell=3000)$. The application of $W_{\mathrm{NR}}^{2}(k)$ thus translates 
into only a small reduction of power (relative to the DM model) at the angular scales most sensitively probed by current small-scale CMB experiments.

The window function measured in the L60CSF simulation has a greater impact on the kSZ power spectrum. The large-scale suppression of the gas density power spectrum seen in Figure 2 produces a significant reduction in kSZ power at all angular scales. At $\ell=3000$, the power is reduced by $1.05 \mu \mathrm{K}^{2}(32 \%)$ and by $1.43 \mu \mathrm{K}^{2}(36 \%)$ at $\ell=10,000$, relative to the NR model. As discussed in the previous section, this reduction in power is primarily driven by the decreased gas density in halos due to overcooling and excessive star-formation. A similar reduction in kSZ power was noted by Trac et al. (2011) when the stellar mass fraction in groups and clusters was increased in their semi-analytic model, reducing the gas fraction by an equivalent amount.

The blue dashed shows the results using the window function measured in the L60CSFz2 model. The amplitude of the power spectrum in this case is close to the mean of the NR and CSF models, with the shape unchanged. A comparison to Figures 11 and 2 provides a clear explanation. Figure 1 demonstrates that the peak of the kSZ contribution comes from spatial scales corresponding to $1 h \mathrm{Mpc}^{-1}<k<3 h \mathrm{Mpc}^{-1}$ at redshift $z \sim 1$. At these scales and redshifts, the L60CSFz2 window function lies almost directly between that of the BolshoiNR and L60CSF simulations, and thus the kSZ power spectrum also lies in between these two cases. The dominant factor determining the amplitude of $\mathrm{kSZ}$ power thus appears to be the mean gas density in halos, which is controlled by the star-formation rate.

We have also investigated the impact of non-linear velocity fluctuations by replacing the linear density power spectrum (i.e. the velocity component of the $\Delta_{R}^{2}$ ) with its non-linear counterpart, as suggested by Zhang et al. (2004). We find that, at $\ell=3000(10,000)$, the amplitude of the kSZ power spectrum in the NR model increases by $1 \%$ (4\%). We have chosen to omit this correction from our fiducial models, as the velocity contribution to $\Delta_{B}^{2}$ in Equation 13 is determined by the gradient of the density field and is therefore curl-free. Hence, it is not clear how one should correctly account for nonlinearities in the velocity field. Nevertheless, the impact on the kSZ power spectrum is likely to be small.

In Figure 6 we plot the relative contribution to the kSZ power spectrum of slices in redshift over the range $0 \leq z \leq z_{\text {rei }}$, at $\ell=3000$. The solid lines show the differential contribution, $d D_{\ell} / d z$, the dashed lines show the cumulative contribution, $D_{\ell}(<z) / D_{\ell}\left(<z_{r e i}\right)$, where $z_{\text {rei }}=10$. The line colors represent our different models, DM (black), NR (red), CSF (blue) and also the OV contribution to the DM model (grey). For comparison, we also plot the results obtained using the L60CSFz2 window function (green).

Comparing the differential redshift contribution of the DM and OV models again demonstrates the enhancement provided by non-linear structure formation at low redshift. Half of the $\mathrm{kSZ}$ signal in the DM model comes from $z<2$, whereas the equivalent fraction for the OV is attained at much higher redshifts $(z \sim 5)$.

The NR model has a similar redshift distribution to the DM model. However, the CSF model predicts a red-

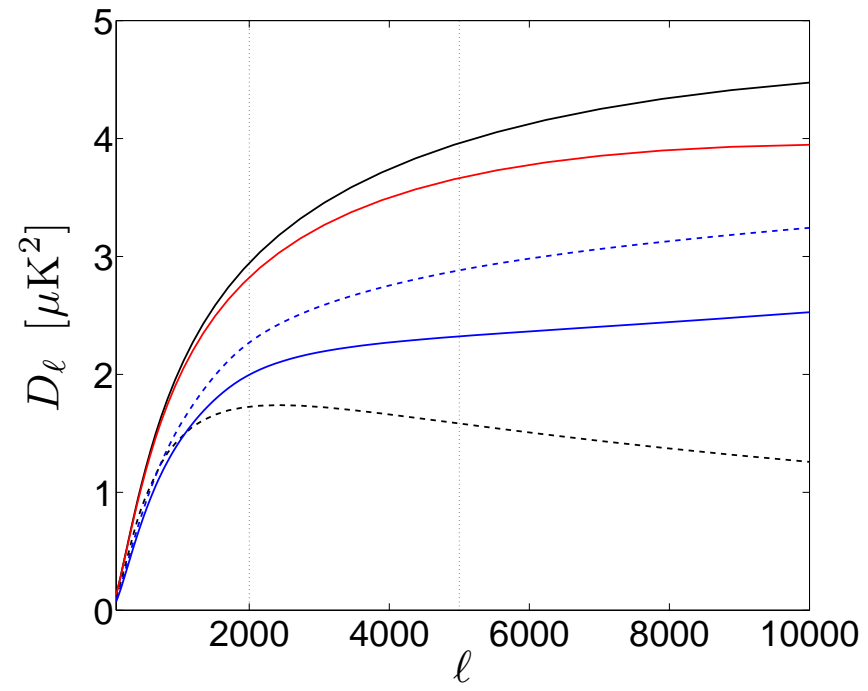

FIG. 5.- The kinetic SZ power spectrum for our three models: DM, NR and CSF (solid black, red and blue lines). The black dashed line shows the linear theory version of the DM model (the Ostriker-Vishniac effect). The blue dashed line shows kSZ power spectrum calculated using the L60CSFz2 window function. The vertical dotted lines denote the approximate range in $\ell$ over which current small-scale CMB experiments such as SPT and ACT are sensitive to $\mathrm{kSZ}$ power.

shift distribution that is significantly flatter. While the differential redshift distribution peaks at the same redshift, the amplitude of the peak is considerably lower. The half-way point of the cumulative distribution is at a much higher redshift, $z \sim 4$, than for the DM and NR models. The right panel of Figure 2 shows that the effects of cooling and star-formation, which reduce the gas density in halos, become increasingly more significant towards lower redshift. This counteracts the boost to the kSZ signal provided by non-linear density fluctuations such that the redshift distribution of the CSF more closely resembles that of the OV model.

The L60CSFz2 simulation, in which radiative cooling is turned-off at $z=1.8$, produces a $\mathrm{kSZ}$ redshift distribution that lies almost exactly in between the NR and CSF models. The distribution diverges from the L60CSF line at $z=1.8$ and then remains at roughly $2 / 3$ the amplitude of the NR model. This is to be expected; once cooling is turned off, gas is no longer being consumed by star-formation. Hence, at lower redshifts, the differences between the L60CSFz2 and the NR model cease to grow. These results suggest that other (and less sudden) variations in the star-formation history would produce a predictable change in the $\mathrm{kSZ}$ redshift distribution: the decrease compared to the NR case would depend on the integrated star-formation rate to that redshift. While it may underestimate the star-formation rate at high redshift (due to the limited mass resolution), the CSF model only diverges significantly from the OV prediction at $z<4$. Given the over-cooling problem at low redshift, it is reasonable to assume that the NR and CSF cases encompass the expected redshift distribution of the kSZ power spectrum at $\ell=3000$.

The redshift distribution of the $\mathrm{kSZ}$ power spectrum can potentially be measured using kSZ tomography (Ho et al. 2009; Shao et al. 2011). The tomography method utilizes catalogues of galaxies with precisely mea- 


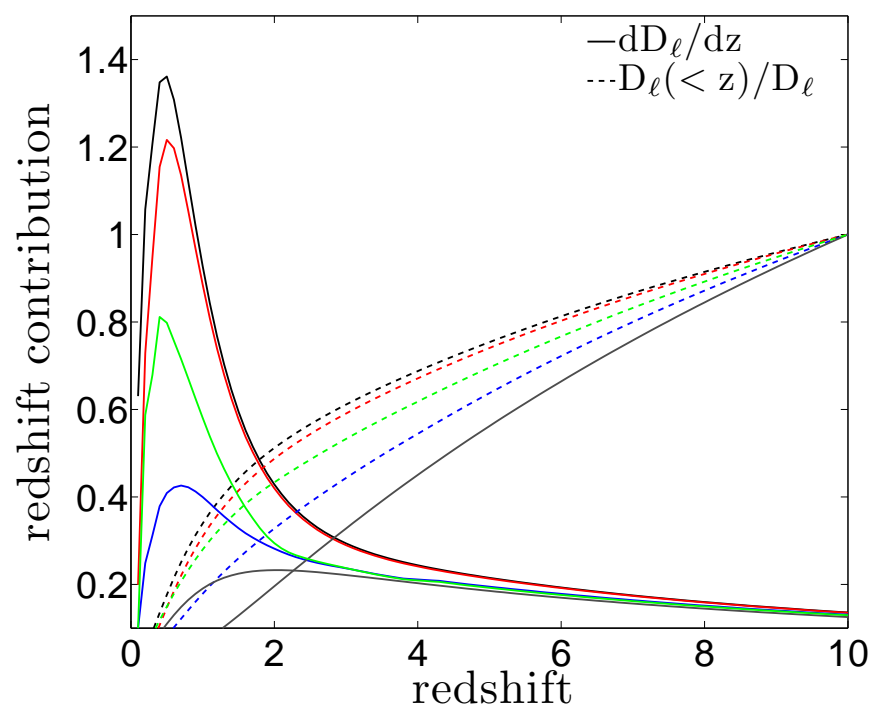

FIG. 6. - The contribution of slices in redshift to the $\mathrm{kSZ}$ power spectrum at $\ell=3000$. The solid lines show the differential contribution, the dashed lines show the cumulative contribution. The line colours represent our different models, DM (black), NR (red), CSF (blue), and the OV contribution to the DM model (grey). Also plotted is the $\mathrm{kSZ}$ redshift distribution predicted by the L60CSFz2 simulation (green)

sured spectroscopic redshifts to reconstruct the largescale velocity field from the 3-D galaxy distribution. When integrated along the line of sight, the product of the reconstructed velocity and density fields provides an estimator for the kinetic SZ temperature fluctuations. This estimator can be broken down into redshift slices and cross-correlated with a map at $\mathrm{CMB}$ frequencies enabling the true $\mathrm{kSZ}$ contribution from within that redshift slice to be measured.

Shao et al. (2011) demonstrate that, by combining Planck with BigBOSS (Schlegel et al. 2011) it will be possible to measure the $\mathrm{kSZ}$ power from the redshift range $0.2 \leq z \leq 0.6$ to better than $10 \%$ precision at $\ell=3000$, and within the range $0.6 \leq z \leq 1$ to better than $20 \%$. Utilizing data from higher angular resolution CMB experiments such as ACT should significantly increase the precision of these measurements. This will not only provide a unique, temperature-independent probe of the inter-galactic medium at lower densities than those typically probed by X-ray and tSZ surveys, but would place tight constraints on the models of the $\mathrm{kSZ}$ power spectrum presented here. Figure 6] demonstrates that much of the theoretical uncertainty on the kSZ signal comes from within the range $0 \leq z \leq 2$; kSZ tomography can provide a powerful probe of the kinetic SZ effect over the lower half of this redshift range.

\subsection{Cosmological scaling}

We have discussed in detail the theoretical uncertainty in the kinetic SZ power spectrum from astrophysical sources. However, the $\mathrm{kSZ}$ signal also scales sensitively with cosmological parameters. There is therefore an additional uncertainty on theoretical estimates of the $\mathrm{kSZ}$ power spectrum due to the precision with which these parameters have been measured. In this section we investigate how the amplitude of the $\mathrm{kSZ}$ power spectrum scales with cosmological parameters - in particular, $\sigma_{8}$ and $\tau-$ and how this scaling varies between our NR and CSF models.

We assume that $D_{\ell}$ scales as a power-law with each cosmological parameter $p$, i.e., $D_{\ell} \propto p^{\alpha}$. We vary each parameter by $20 \%$ of its fiducial value (holding the other parameters fixed) and measure the value of $\alpha$. We have verified that the power-law scaling is appropriate for all parameters. The results are presented in Tables 2 and 3 . We give the results over the angular multipole number range $1000<\ell<10,000$. Also given are the bandpowers, $D_{\ell}$, for each model.

The $\mathrm{kSZ}$ power spectrum amplitude is most sensitive to $\sigma_{8}$, scaling as $\sigma_{8}^{4.3-5.1}$ from $\ell=1000$ to 10,000 (NR model). This scaling is simple to understand. The $\mathrm{kSZ}$ power spectrum involves the product of the density and velocity power spectra; in the linear regime each contributes a power of 2 to the scaling with $\sigma_{8}$. The OV power spectrum thus scales exactly as $\sigma_{8}^{4}$. Non-linear corrections to the matter power spectrum steepen the $\sigma_{8}$ dependence. This steepening is related to the fraction of the $\mathrm{kSZ}$ signal that originates at low redshift where the non-linear corrections have grown large. Figure 6] shows that the maximal contribution to the OV signal comes from $z=2$, whereas the full $\mathrm{kSZ}$ signal for the DM model peaks at $z=0.5$. This shift in the redshift contribution is driven by non-linear structure formation, which explains the steeper $\sigma_{8}$ dependence. The same argument applies to the difference in the $\sigma_{8}$ scaling between the NR and CSF models; a smaller fraction of the signal derives from low redshift in the latter and so the scaling with $\sigma_{8}$ is weaker. We note that changing $\sigma_{8}$ principally varies the overall amplitude of the $\mathrm{kSZ}$ signal; while the scaling does have some $\ell$-dependence, the impact on the shape of the power spectrum is small over the parameter space explored.

Some of the tightest constraints on $\sigma_{8}$ to date have been obtained from measurements of the primary CMB power spectrum and the abundance of galaxy clusters. For example, Vikhlinin et al. (2009) used a sample of 86 clusters to obtain $\sigma_{8}\left(\Omega_{M} / 0.25\right)^{0.47}=0.81 \pm 0.01 \pm 0.02$ (statistical and systematic errors, respectively). Using the CSF model scaling, this translates to an uncertainty of approximately $\pm 15 \%$ on the $\mathrm{kSZ}$ power spectrum at $\ell=3000$. This is equivalent to the uncertainty in $D_{\ell}$ provided by baryon physics presented in the previous section.

The magnitude of the $\mathrm{kSZ}$ signal is also sensitive to the optical depth to reionization, $\tau$, which is directly related to the redshift at which reionization occurs (see Equation 2). In Tables 2 and 3 we give the scaling of $\mathcal{D}_{\ell}$ with both $\tau$ and $z_{\text {rei }}$. The CSF model has a greater sensitivity to each of these parameters as a slightly larger fraction of the signal is generated at high redshift. In principle, the post-reionization $\mathrm{kSZ}$ power spectrum could be used to measure $z_{\text {rei }}$. In practice, the uncertainty on other cosmological parameters washes out this information.

To this point we have ignored the effect of helium reionization. The amplitude of the $\mathrm{kSZ}$ signal also scales as the square of the ionization fraction, $\chi^{2}$, which is dependent on the ionization state of helium. Our fiducial model assumes that helium remains neutral at all epochs. If, instead, we assume that helium is singly or doubly ionized at $z_{\text {rei }}$ then the amplitude of the $\mathrm{kSZ}$ power spectrum 
will increase by a ( $\ell$-independent) factor of 1.16 or 1.33 , due to the increase in the free electron density. A more realistic model in which helium is singly ionized at $z=6$ and doubly ionized at $z=3$ (e.g. Furlanetto \& Oh 2008) would increase the $\mathrm{kSZ}$ power by a factor of $1.22(1.26)$ at $\ell=3000(10,000)$ relative to our baseline, neutral helium, model. Hence, the level of uncertainty on the $\mathrm{kSZ}$ power spectrum due to Helium reionization is equivalent to that due to the uncertainty on $\sigma_{8}$.

TABLE 2

KSZ Cosmological ScAling: NR

\begin{tabular}{c|c|c|c|c|c|c}
\hline \hline$\ell$ & $\mathcal{D}_{\ell}$ & $H_{0}$ & $\sigma_{8}$ & $\Omega_{b}$ & $z_{\text {rei }}$ & $\tau$ \\
& $\mu \mathrm{K}^{2}$ & $70 \mathrm{~km} / \mathrm{s} / \mathrm{Mpc}$ & 0.82 & 0.047 & 10.0 & 0.076 \\
\hline 1000 & 1.97 & 1.18 & 4.31 & 2.29 & 0.46 & 0.32 \\
2000 & 2.82 & 1.51 & 4.48 & 2.18 & 0.46 & 0.32 \\
3000 & 3.24 & 1.68 & 4.62 & 2.13 & 0.43 & 0.30 \\
4000 & 3.49 & 1.80 & 4.73 & 2.10 & 0.40 & 0.28 \\
5000 & 3.66 & 1.88 & 4.81 & 2.08 & 0.37 & 0.26 \\
6000 & 3.77 & 1.95 & 4.88 & 2.06 & 0.34 & 0.24 \\
7000 & 3.85 & 2.00 & 4.94 & 2.05 & 0.32 & 0.22 \\
8000 & 3.90 & 2.05 & 4.99 & 2.03 & 0.30 & 0.21 \\
9000 & 3.93 & 2.09 & 5.04 & 2.02 & 0.29 & 0.20 \\
10000 & 3.95 & 2.13 & 5.07 & 2.01 & 0.28 & 0.19 \\
\hline
\end{tabular}

Note. - The scaling $\alpha$ of the $\mathrm{kSZ}$ power spectrum with cosmological parameters, $p$, for the NR model, where we assume $D_{\ell} \propto p^{\alpha}$. The fiducial parameters are given in the second line and the corresponding values of $\mathcal{D}_{\ell}$ in the second column.

TABLE 3

KSZ Cosmological Scaling: CSF

\begin{tabular}{c|c|c|c|c|c|c}
\hline \hline$\ell$ & $\mathcal{D}_{\ell}$ & $H_{0}$ & $\sigma_{8}$ & $\Omega_{b}$ & $z_{\text {rei }}$ & $\tau$ \\
& $\mu \mathrm{K}^{2}$ & $70 \mathrm{~km} / \mathrm{s} / \mathrm{Mpc}$ & 0.82 & 0.047 & 10.0 & 0.076 \\
\hline 1000 & 1.43 & 1.09 & 4.19 & 2.31 & 0.63 & 0.43 \\
2000 & 2.00 & 1.46 & 4.33 & 2.18 & 0.66 & 0.45 \\
3000 & 2.19 & 1.65 & 4.46 & 2.12 & 0.64 & 0.44 \\
4000 & 2.27 & 1.78 & 4.57 & 2.09 & 0.60 & 0.41 \\
5000 & 2.32 & 1.87 & 4.67 & 2.06 & 0.55 & 0.38 \\
6000 & 2.36 & 1.94 & 4.76 & 2.04 & 0.52 & 0.35 \\
7000 & 2.40 & 2.00 & 4.83 & 2.02 & 0.48 & 0.33 \\
8000 & 2.44 & 2.06 & 4.89 & 2.01 & 0.45 & 0.31 \\
9000 & 2.48 & 2.10 & 4.95 & 2.00 & 0.42 & 0.29 \\
10000 & 2.52 & 2.14 & 4.99 & 1.99 & 0.40 & 0.27 \\
\hline
\end{tabular}

Note. - Similar to Table 2 but for the CSF model.

\subsection{Comparisons with Simulations}

A number of previous studies have used the output of cosmological simulations to make predictions for the shape and amplitude of the kinetic SZ power spectrum (da Silva et al. 2001; White et al. 2002; Hallman et al. 2009; Trac et al. 2011; Battaglia et al. 2010). Rather than calibrating an analytic model to their simulations (as we have done), these studies generate mock sky maps of the temperature fluctuations sourced by the kinetic SZ effect. Maps are typically constructed by stacking outputs of the simulated volume over a range of timesteps, often rotating or translating each output to prevent the repetition of structures along the line of sight and generate a larger simulated sky area.

Comparing our model predictions with those made from synthetic sky maps is a non-trivial task. The simulations encompass a range of cosmological parameters and often have a more limited redshift range than our fiducial model (where $z_{\text {rei }}=10$; for simplicity we refer to the highest redshift output of each simulation as ' $z_{\text {rei }}$ '). Furthermore, as we described in detail in Section 4. the limited size of the simulation volume results in the truncation of large-scale velocity modes and thus a significant underestimate of the $\mathrm{kSZ}$ signal. For example, we estimate that a simulation box of side length 100 $\mathrm{Mpc} / h$ would underestimate the $\mathrm{kSZ}$ power at $\ell=3000$ by $60 \%$. A box size of at least $1 \mathrm{Gpc} / h$ is required to fully account (to within $1 \%$ of the total power) for all the velocity modes that contribute to the $\mathrm{kSZ}$ power spectrum at $\ell=3000$.

To compare our model with that of previous studies, we must correct their results to account for the limited simulation box size, the variations in the maximum redshift assumed, and differing cosmological parameters. We use our NR model as a baseline to rescale the simulation results to our fiducial cosmology (namely, $\sigma_{8}=0.82$, $\Omega_{b}=0.047$ and $\left.z_{\text {rei }}=10\right)$, and to estimate the amount by which the kSZ amplitude must be increased to account for velocity mode-truncation. We also include a helium correction such that the level of helium ionization is consistent with our fiducial model (i.e., neutral at all epochs).

The predictions for the $\mathrm{kSZ}$ effect from simulations presented in previous work are shown in Table 4. The table shows three columns for the $\mathrm{kSZ}$ power at $\ell=3000$ $\left(\mathcal{D}_{3000}\right)$, the first shows the raw prediction for $\mathcal{D}_{3000}$ taken from each work. The second column shows the $\mathrm{kSZ}$ power rescaled to our fiducial cosmology using the scalings given in Table 2. The third column shows $\mathcal{D}_{3000}$ having additionally corrected for the simulation box size in each case.

We consider the results from the smoothed-particlehydrodynamics (SPH) simulations of White et al. (2002, henceforth, WHS02), the Eulerian ENZO simulations of Hallman et al. (2009, H09) and both the non-radiative (NR) simulations and those including cooling, starformation and active galactic nucleus (AGN) feedback performed by Battaglia et al. (2010, B10). The WHS02 simulation includes radiative cooling, star-formation and galactic winds (although they note these processes may be inhibited by the limited mass resolution of their simulation). The H09 simulation is run in the non-radiative regime. We also include the 'adiabatic' and 'standard' semi-analytic models of Trac et al. (2011, TBO11) (see also Sehgal et al. 2010). The adiabatic model assumes that gas resides in hydrostatic equilibrium in the potential well of dark matter halos identified in their N-body simulation. The standard model also includes simple prescriptions for star-formation and non-gravitational energy feedback from supernovae and AGN.

The cosmological rescaling for the WHS02 and H09 simulations are fairly large as both these simulations were run assuming $\sigma_{8}=0.9$ and $z_{\text {rei }}=19$ and 3 , respectively. 
The box-size corrections are largest for WHS02, who used a $100 \mathrm{Mpc} / \mathrm{h}$ box, and B10, who generated their kSZ predictions using simulation box sizes of $165 \mathrm{Mpc} / h$.

In general, the simulations predict between 2.27 and $3.91 \mu \mathrm{K}^{2}$, depending on the level of gas physics included, although H09 measure an amplitude of more than twice the upper end of this range. They are therefore consistent with the predictions of our NR $\left(3.2 \mu \mathrm{K}^{2}\right)$ and CSF $\left(2.2 \mu \mathrm{K}^{2}\right)$ models. B10 find that the inclusion of radiative cooling, star-formation, and AGN feedback reduces the $\mathrm{kSZ}$ power by $1 \mu \mathrm{K}^{2}$. The semi-analytic models of TBO11 lie on the low end of the range of simulations discussed here. Their 'adiabatic' model is similar to our NR model, while their 'standard' model includes prescriptions for star-formation and energy feedback. The effect of these prescriptions is to reduce the $\mathrm{kSZ}$ signal by $0.4 \mu \mathrm{K}^{2}$. They also explore the $\mathrm{kSZ}$ signal obtained when the stellar mass in groups and clusters was increased beyond that of the standard model, finding a further reduction of $0.3 \mu \mathrm{K}^{2}$. This supports our assertion that the CSF model - which predicts the lowest amplitude of all provides a robust lower limit on the $\mathrm{kSZ}$ power spectrum due to the reduction of the mean gas density in groups and clusters via star-formation.

TABLE 4

KSZ PRedictions From Simulations

\begin{tabular}{c|c|c|c}
\hline \hline Paper & \multicolumn{3}{|c}{$\mathcal{D}_{3000}\left[\mu \mathrm{K}^{2}\right]$} \\
& Unnorm. & Cosm Cor. & Box Cor. \\
\hline WHS02 & 3.00 & 1.57 & 3.91 \\
H09 & 7.40 & 8.50 & 9.45 \\
B10, NR & 2.50 & 2.42 & 4.03 \\
B10, AGN & 1.50 & 1.45 & 2.42 \\
TBO11, adiabatic & 2.50 & 2.70 & 2.70 \\
TBO11, standard & 2.10 & 2.27 & 2.27 \\
This work, DM & $\ldots$ & $\ldots$ & 3.44 \\
This work, NR & $\ldots$ & $\ldots$ & 3.24 \\
This work, CSF & $\ldots$ & $\ldots$ & 2.19 \\
\hline
\end{tabular}

Note. - The amplitude of the kSZ power predicted by hydrodynamical simulations in previous work. We show the results from the SPH simulations of White et al. (2002, WHS02), the ENZO simulations of Hallman et al. (2009, $\mathrm{H} 09$ ), both the non-radiative (NR) simulations and those including cooling, star-formation and AGN feedback from Battaglia et al. (2010, B10) and the 'adiabatic' and 'standard' models from the N-body plus semi-analytic approach of Trac et al. (2011, TBO11).

\subsection{Comparisons with Observations}

To date, the kinetic SZ effect has not yet been detected observationally. However, both the Atacama Cosmology Telescope and the South Pole Telescope have placed upper limits on the amplitude of the $\mathrm{kSZ}$ power at $\ell=3000$. From $296 \mathrm{deg}^{2}$ of data, Dunkley et al. (2011) obtained a $2 \sigma$ upper limit of $D_{\ell}=8 \mu \mathrm{K}^{2}$. More recently Shirokoff et al. (2011) obtained an upper limit of $D_{\ell}=6.5 \mu \mathrm{K}^{2}$ (also $2 \sigma$ ) from $210 \mathrm{deg}^{2}$. Therefore, observations do not currently constrain any of the models that we have presented in this work, although the pre- dictions of the simulations of Hallman et al. (2009) are inconsistent with the most recent measurements.

The main difficulty in measuring $\mathrm{kSZ}$ power lies with disentangling the signal from both the thermal SZ effect and bright foregrounds, primarily dusty, star-forming galaxies (DSFG). Unfortunately, the angular shape of both the tSZ and DSFG power spectra is similar to that of the kSZ signal, so it is difficult to separate these signals in $\ell$-space. However, each has a very different frequency dependence; multifrequency observations will be extremely effective in separating these components. Combining SPT or ACT data with Planck and Herschel should enable a significant detection of $\mathrm{kSZ}$ power in the near future.

\section{DISCUSSION \& CONCLUSION}

The kinetic SZ (kSZ) power spectrum is generated by the coupling between large scale velocity flows and smallscale density perturbations. To predict its amplitude and shape it is necessary to understand the behavior of the power spectrum of gas density fluctuations over the range of scales corresponding to $0.1 \leq k \leq 10 h \mathrm{Mpc}^{-1}$. In this work, we have introduced a new model for the kinetic SZ power spectrum that accounts for the effect of baryonic physics on the power spectrum of gas density fluctuations and thus on the kSZ power spectrum.

To this end, we defined a window function, $W^{2}(k)=$ $P_{\text {gas }}(k) / P_{\mathrm{DM}}(k)$, to provide a mapping between dark matter and gas density power spectra in our calculations. We utilized hydrodynamic simulations - run in both the non-radiative regime and including radiative cooling and star-formation - to measure the window functions and investigated their effect on the $\mathrm{kSZ}$ power spectrum. We have presented three models for the $\mathrm{kSZ}$ power spectrum: DM (dark matter), in which gas density fluctuations follow those of the dark matter at all scales, NR (non-radiative) and CSF (cooling \& star-formation) in which we use the window functions measured in our hydrodynamic simulations.

There is only a small difference between the DM and NR models. Gas density fluctuations in our non-radiative simulations are suppressed at spatial scales smaller than those that contribute significantly to the $\mathrm{kSZ}$ power spectrum (for $\ell<10,000$ ). At $\ell=3000$, the NR model predicts $\mathcal{D}_{\ell}=3.24 \mu \mathrm{K}^{2}$, only $0.20 \mu \mathrm{K}^{2}$ below the DM model. However, the CSF power spectrum has a significantly lower amplitude as well as a flatter shape than either the NR or DM models. At $\ell=3000$, the CSF model predicts $\mathcal{D}_{\ell}=2.19 \mu \mathrm{K}^{2}, 1.25 \mu \mathrm{K}^{2}$ below the DM model. The reduction in power is driven by the decrease in the mean gas density in group- and cluster-mass halos due to the high-levels of cooling and star-formation in our simulation. This in turn reduces the amplitude of the gas density power spectrum and thus the $\mathrm{kSZ}$ power spectrum.

To investigate the impact on the shape and amplitude of the $\mathrm{kSZ}$ power spectrum of variations in star-formation history, we rerun our CSF simulation having turned off radiative cooling at $z=1.8$. We find that the resulting window function smoothly evolved from a CSF-like shape to a NR-like shape, with the amplitude (at large scales / low $k$ ) lying midway between the two cases. The resulting $\mathrm{kSZ}$ power spectrum was thus also almost directly between that of the NR and CSF models. 
We have argued that our NR and CSF models provide reasonable upper and lower limits to the effect of astrophysical processes on the kSZ power spectrum. The NR model is calibrated from a simulation that included no cooling and star-formation and thus no means of reducing the gas density. On the other hand, our CSF model is calibrated to a simulation that suffers from the over-cooling problem, i.e., from excessive cooling and star-formation. Hence, we expect our CSF model to underestimate the true kSZ signal. Nevertheless, the difference between the NR and CSF models at $\ell=3000$ is only $1 \mu \mathrm{K}^{2}$. Taking the mean of these two models, we find that the astrophysical uncertainty on the kSZ power spectrum amplitude is roughly $\pm 20 \%$. This is significantly less than that of the thermal SZ power spectrum, for which the current theoretical uncertainty on the amplitude is $\pm 50 \%$ (Shaw et al. 2010; Trac et al. 2011).

There are two caveats to this argument. Firstly, our fiducial models assume helium remains neutral at all epochs. Singly or doubly ionized helium would increase the amplitude of our models by up to $33 \%$, depending on the redshifts at which helium reionization occurs. Secondly, the amplitude of the $\mathrm{kSZ}$ power spectrum is sensitive to cosmological parameters, namely $\sigma_{8}, \Omega_{b}, H_{0}$ and $\tau$ (and, equivalently, $z_{\text {rei }}$ ). We have investigated the scaling of our models with cosmological parameters, finding that $\mathcal{D}_{3000} \propto \sigma_{8}^{4.5} \Omega_{b}^{2.1} H_{0}^{1.7} \tau^{0.44}$ for the CSF model. The current $1 \sigma$ uncertainty on $\sigma_{8}$ obtained from cluster number counts (Vikhlinin et al. 2009) translates to a $\pm 15 \%$ uncertainty on $\mathcal{D}_{3000}$.

We have compared our models with predictions made directly from hydrodynamic simulations (White et al. 2002; Hallman et al. 2009; Battaglia et al. 2010; Trac et al. 2011). These studies measured the kSZ power spectrum using synthetic kSZ maps constructed by the stacking of simulation box outputs. A serious drawback of this approach is that velocity modes are truncated at the scale of the simulation box. For boxes of side length $\ll 1 \mathrm{Gpc} / h$, this can result in a significant underestimate of the $\mathrm{kSZ}$ signal. We used our model to correct the predictions of the simulations in previous studies for this effect, as well as differences in cosmological parameters. We then found a reasonably good agreement among different simulations results and the range predicted by our models.

In this work we consider only the homogeneous- or post-reionization contribution to the kinetic SZ signal. It is well known that models of inhomogeneous reionization, in which different regions of the Universe are reionized at different times, predict a patchy kinetic SZ signal. Zahn et al. (2005) and McQuinn et al. (2005) demonstrate that, to first order, the magnitude of the patchy signal is dependent on the duration of reionization. Given current technology, the kinetic SZ power spectrum thus provides a unique probe of the redshift range spanned by the epoch of reionization. However, current observations only provide a measure of the sum of the patchy and post-reionization kSZ power spectra at angular scales around $\ell=3000$. Therefore, in order to extract the patchy component, it is important to have a good theoretical understanding of the post-reionization contribution. In this work we have performed a detailed investigation of the theoretical uncertainty on the postreionization $\mathrm{kSZ}$ power spectrum due to astrophysical processes, cosmological parameters and helium reionization. Adding these in quadrature produces a total uncertainty of $\sim 30 \%$ on the amplitude of the post-reionization $\mathrm{kSZ}$ power spectrum at $\ell=3000$.

Improvements in our understanding of the $\mathrm{kSZ}$ power spectrum can be made both theoretically and observationally. Models and simulations of the kinetic SZ power spectrum can be further developed to make improved theoretical predictions. The over-cooling problem may be mitigated by the inclusion of energy feedback from active galactic nuclei in our simulations (Siiacki et al. 2007; Booth \& Schave 2009; Battaglia et al. 2010; McCarthy et al. 2010; Tevssier et al. 2011). Feedback from AGN can heat gas sufficiently in high density regions to slow the local cooling (and thus star-formation) rate. da Silva et al. (2001) demonstrated that the inclusion of a preheating model to their simulations suppressed star-formation and increased the kSZ power with respect to simulations without preheating. On the other hand Battaglia et al. (2010) also demonstrate that AGN also heat gas out to large cluster radii, flattening the density profile, reducing the gas fraction in groups and clusters. The net impact of AGN feedback on the SZ power spectrum will depend on the extent to which these two effects balance one another. Recent models of AGN in simulations have typically been tuned to match local $(z \sim 0)$ observations, however, as we have demonstrated, a significant contribution of the kSZ comes from larger redshifts where the star formation histories of halos are relatively unconstrained. Furthermore, higher (sub-kpc) resolution simulations may be required to resolve starformation in lower mass halos.

From an observational perspective, small-scale CMB experiments have currently only placed upper limits on the amplitude of the kSZ power spectrum and do not constrain any of our models (Shirokoff et al. 2011; Dunkley et al. 2011). However, with increasing area and frequency coverage, observations will become significantly more constraining in the near future. kSZ tomography has the potential to constrain models of the postreionization signal by measuring its redshift dependence. This can be achieved by cross-correlating a kSZ estimator constructed from spectroscopic galaxy catalogues with a CMB map (Ho et al. 2009; Shao et al. 2011). We have investigated the contribution of slices in redshift to the total kinetic SZ power for each of our models. We found that the principle difference between the NR and CSF models comes from sources in the range $0 \leq z \leq 2$. Upcoming surveys have the potential to measure the redshift distribution of the $\mathrm{kSZ}$ effect out to $z \sim 1$ using kSZ tomography. This therefore provides a potentially powerful means of constraining our kSZ power spectrum models.

\section{ACKNOWLEDGMENTS}

We would like to thank Ryan Keisler, Christian Reichardt, Oliver Zhan, Gil Holder, Wayne Hu, Nikhil Padmanabhan, Pengjie Zhang and Zheng Zheng for useful and informative discussions. This work was supported in part by the NSF grant AST-1009811, by NASA ATP grant NNX11AE07G, by the Yale University, and by the facilities and staff of the Yale University Faculty of Arts and Sciences High Performance Computing Center. D.H.R. acknowledges the support of NSF grant OCI- 
0904484. The simulations used in this study were performed on the SGI Altix system (columbia) at NASA Ames and the BulldogM cluster at the Yale University
Faculty of Arts and Sciences High Performance Computing Center.

\section{REFERENCES}

Atrio-Barandela, F., Mücket, J. P., \& Génova-Santos, R. 2008, ApJ, 674, L61

Battaglia, N., Bond, J. R., Pfrommer, C., Sievers, J. L., \& Sijacki, D. 2010, ApJ, 725, 91

Becker, G. D., Rauch, M., \& Sargent, W. L. W. 2007, ApJ, 662, 72

Blumenthal, G. R., Faber, S. M., Flores, R., \& Primack, J. R. 1986, ApJ, 301, 27

Bolton, J. S. \& Haehnelt, M. G. 2007, MNRAS, 381, L35

Booth, C. M. \& Schaye, J. 2009, MNRAS, 398, 53

Bryan, G. L. \& Norman, M. L. 1998, ApJ, 495, 80

Casarini, L., Macciò, A. V., Bonometto, S. A., \& Stinson, G. S. 2011, MNRAS, 412, 911

da Silva, A. C., Kay, S. T., Liddle, A. R., Thomas, P. A., Pearce,

F. R., \& Barbosa, D. 2001, ApJ, 561, L15

Dodelson, S. \& Jubas, J. M. 1995, ApJ, 439, 503

Duffy, A. R., Schaye, J., Kay, S. T., Dalla Vecchia, C., Battye, R. A., \& Booth, C. M. 2010, MNRAS, 405, 2161

Dunkley, J., Hlozek, R., Sievers, J., \& et al. 2011, ApJ, 739, 52

Fan, X., Strauss, M. A., Becker, R. H., White, R. L., Gunn, J. E. Knapp, G. R., Richards, G. T., Schneider, D. P., Brinkmann, J., \& Fukugita, M. 2006, AJ, 132, 117

Ferland, G. J., Korista, K. T., Verner, D. A., Ferguson, J. W. Kingdon, J. B., \& Verner, E. M. 1998, PASP, 110, 761

Furlanetto, S. R. \& Oh, S. P. 2008, ApJ, 681, 1

Giodini, S., Pierini, D., Finoguenov, A., \& et al. 2009, ApJ, 703, 982

Gnedin, N. Y., Baker, E. J., Bethell, T. J., Drosback, M. M., Harford, A. G., Hicks, A. K., Jensen, A. G., Keeney, B. A., Kelso, C. M., Neyrinck, M. C., Pollack, S. E., \& van Vliet, T. P. 2003, ApJ, 583, 525

Gnedin, N. Y. \& Hui, L. 1998, MNRAS, 296, 44

Gnedin, O. Y., Ceverino, D., Gnedin, N. Y., Klypin, A. A., Kravtsov, A. V., Levine, R., Nagai, D., \& Yepes, G. 2011, ArXiv e-prints

Gnedin, O. Y., Kravtsov, A. V., Klypin, A. A., \& Nagai, D. 2004 ApJ, 616, 16

Gonzalez, A. H., Zaritsky, D., \& Zabludoff, A. I. 2007, ApJ, 666, 147

Gruzinov, A. \& Hu, W. 1998, ApJ, 508, 435

Haardt, F. \& Madau, P. 1996, ApJ, 461, 20

Hallman, E. J., O'Shea, B. W., Smith, B. D., Burns, J. O., \& Norman, M. L. 2009, ApJ, 698, 1795

Heitmann, K., White, M., Wagner, C., Habib, S., \& Higdon, D. 2010, ApJ, 715, 104

Ho, S., Dedeo, S., \& Spergel, D. 2009, ArXiv e-prints

Hu, W. 2000, ApJ, 529, 12

Iliev, I. T., Pen, U.-L., Bond, J. R., Mellema, G., \& Shapiro, P. R. 2007, ApJ, 660, 933

Jaffe, A. H. \& Kamionkowski, M. 1998, Phys. Rev. D, 58, 043001

Jing, Y. P., Zhang, P., Lin, W. P., Gao, L., \& Springel, V. 2006, ApJ, 640, L119

Keisler, R., Reichardt, C. L., \& et al. 2011, ApJ, 743, 28

Klypin, A., Kravtsov, A. V., Bullock, J. S., \& Primack, J. R. 2001, ApJ, 554, 903

Knox, L., Scoccimarro, R., \& Dodelson, S. 1998, Physical Review Letters, 81, 2004

Kosowsky, A. 2003, New Astronomy Review, 47, 939

Kravtsov, A., Gonzalez, A., Vikhlinin, A., \& et al. 2009, in Astronomy, Vol. 2010, astro2010: The Astronomy and Astrophysics Decadal Survey, 164-+

Kravtsov, A. V., Klypin, A., \& Hoffman, Y. 2002, ApJ, 571, 563

Kravtsov, A. V., Nagai, D., \& Vikhlinin, A. A. 2005, ApJ, 625, 588

Lidz, A., Oh, S. P., \& Furlanetto, S. R. 2006, ApJ, 639, L47

Lueker, M., Reichardt, C. L., Schaffer, K. K., , \& et al. 2010, ApJ, 719, 1045

Ma, C.-P. \& Fry, J. N. 2002, Physical Review Letters, 88, 211301
Marriage, T. A., Acquaviva, V., Ade, P. A. R., \& et al. 2011, ApJ, 737, 61

Marrone, D. P., Smith, G. P., Okabe, N., \& et al. 2011, ArXiv e-prints

McCarthy, I. G., Schaye, J., Ponman, T. J., Bower, R. G., Booth, C. M., Dalla Vecchia, C., Crain, R. A., Springel, V., Theuns, T., \& Wiersma, R. P. C. 2010, MNRAS, 406, 822

McQuinn, M., Furlanetto, S. R., Hernquist, L., Zahn, O., \& Zaldarriaga, M. 2005, ApJ, 630, 643

Mortlock, D. J., Warren, S. J., \& et al. 2011, Nature, 474, 616

Navarro, J. F., Frenk, C. S., \& White, S. D. M. 1997, ApJ, 490, 493

Oh, S. P. \& Furlanetto, S. R. 2005, ApJ, 620, L9

Ostriker, J. P. \& Vishniac, E. T. 1986, ApJ, 306, L51

Ouchi, M., Shimasaku, K., Furusawa, H., \& et al. 2010, ApJ, 723, 869

Planck Collaboration, Ade, P. A. R., Aghanim, N., Arnaud, M., Ashdown, M., Aumont, J., Baccigalupi, C., Balbi, A., Banday, A. J., Barreiro, R. B., \& et al. 2011, ArXiv e-prints

Rudd, D. H., Zentner, A. R., \& Kravtsov, A. V. 2008, ApJ, 672

Ruhl, J., Ade, P. A. R., Carlstrom, J. E., \& et al. 2004, in Society of Photo-Optical Instrumentation Engineers (SPIE) Conference Series, Vol. 5498, Society of Photo-Optical Instrumentation Engineers (SPIE) Conference Series, ed. C. M. Bradford, P. A. R. Ade, J. E. Aguirre, J. J. Bock, M. Dragovan, L. Duband, L. Earle, J. Glenn, H. Matsuhara, B. J. Naylor, H. T. Nguyen, M. Yun, \& J. Zmuidzinas, 11-29

Schlegel, D., Abdalla, F., Abraham, T., Ahn, C., \& et al. 2011, ArXiv e-prints

Sehgal, N., Bode, P., Das, S., Hernandez-Monteagudo, C., Huffenberger, K., Lin, Y., Ostriker, J. P., \& Trac, H. 2010, ApJ, 709, 920

Semboloni, E., Hoekstra, H., Schaye, J., van Daalen, M. P., \& McCarthy, I. G. 2011, MNRAS, 417, 2020

Shao, J., Zhang, P., Lin, W., Jing, Y., \& Pan, J. 2011, MNRAS, 413,628

Shaw, L. D., Nagai, D., Bhattacharya, S., \& Lau, E. T. 2010 ApJ, 725, 1452

Shirokoff, E., Reichardt, C. L., Shaw, L., Millea, M., \& et al. 2011, ApJ, 736, 61

Sijacki, D., Springel, V., Di Matteo, T., \& Hernquist, L. 2007, MNRAS, 380, 877

Smith, R. E., Peacock, J. A., Jenkins, A., White, S. D. M., Frenk, C. S., Pearce, F. R., Thomas, P. A., Efstathiou, G., \& Couchman, H. M. P. 2003, MNRAS, 341, 1311

Springel, V. \& Hernquist, L. 2003, MNRAS, 339, 312

Teyssier, R., Moore, B., Martizzi, D., Dubois, Y., \& Mayer, L. 2011, MNRAS, 414, 195

Trac, H., Bode, P., \& Ostriker, J. P. 2011, ApJ, 727, 94

van Daalen, M. P., Schaye, J., Booth, C. M., \& Dalla Vecchia, C. 2011, MNRAS, 1079

Vanderlinde, K., Crawford, T. M., de Haan, T., Dudley, J. P., Shaw, L., \& et al. 2010, ApJ, 722, 1180

Vikhlinin, A., Kravtsov, A. V., Burenin, R. A., Ebeling, H., Forman, W. R., Hornstrup, A., Jones, C., Murray, S. S., Nagai, D., Quintana, H., \& Voevodkin, A. 2009, ApJ, 692, 1060

Vishniac, E. T. 1987, ApJ, 322, 597

White, M., Hernquist, L., \& Springel, V. 2002, ApJ, 579, 16

Williamson, R., Benson, B. A., High, F. W., Vanderlinde, K., \& et al. 2011, ApJ, 738, 139

Zahn, O., Reichardt, C. L., Shaw, L., \& et al. 2011, ArXiv e-prints

Zahn, O., Zaldarriaga, M., Hernquist, L., \& McQuinn, M. 2005, ApJ, 630, 657

Zhang, P., Pen, U.-L., \& Trac, H. 2004, MNRAS, 347, 1224 